\title{
Epidermolysis bullosa hereditaria
}

\section{Epidermolysis Bullosa Hereditaria}

M. Laimer, J. W. Bauer, C. M. Lanschützer, E. Nischler, H. Hintner

Universitätsklinik für Dermatologie, Paracelsus Medizinische Privatuniversität (PMU) Salzburg, Salzburger Landeskliniken (SALK)

\section{Lernziele}

$\nabla$

Kenntnisse über:

- Definition der Epidermolysis bullosa hereditaria

- Klassifikation

- spezielle klinische Manifestationen

- wichtige Komplikationen

- Diagnose

- Therapie

\section{Definition}

\section{$\nabla$}

Der Begriff „Epidermolysis bullosa (EB) hereditaria“ umfasst eine Gruppe seltener $(=$ rare $=$ orphan disease), heterogener, genetisch bedingter Hauterkrankungen, welchen charakteristischerweise Mutationen in strukturell wie auch funktionell wichtigen Proteinen der dermo-epidermalen Basalmembranzone (BMZ) oder des Zytoskleletts von Keratinozyten zugrunde liegen (๑ Abb. 1).

Mutationen in den Genen für Strukturproteine von Keratinozyten oder der dermo-epidermalen Basalmembranzone sind die Ursache für die mukokutane Blasen- und Erosionsbildung bei der erblichen Epidermolysis bullosa, die durch zahlreiche Extrakutanmanifestationen zur Systemerkrankung werden kann.

Durch das folgende Fehlen oder den Funktionsverlust der Strukturproteine kommt es zum Verlust der mechanischen Integrität und -stabilität der dermo-epidermalen Mikroarchitektur und zu erhöhter Verletzlichkeit, die bereits nach geringen Traumata (oft nur eine stärkere Berührung) oder sogar spontan (oft bei hoher Außentemperatur) zur Spaltbildung und Entstehung von mukokutanen Blasen und Erosionen führt (Synonym: „mechanobullöse“ Dermatosen). Zudem kann das Auftreten von mannigfaltigen Extrakutanmanifestationen die EB zu einer System- krankheit mit signifikanter Morbidität und Mortalität werden lassen.

Die hohe genetische Variabilität bedingt ein breites Spektrum an klinischen Manifestationen.

Bisher konnten mehr als 10 Gene identifiziert werden, die durch Sequenzänderung zum Fehlen, zur Reduktion oder zur Bildung funktionsuntüchtiger Strukturproteine in Keratinozyten oder innerhalb der dermo-epidermalen Junktionszone führen. Die hohe Zahl an betroffenen Genen, deren unterschiedliche funktionelle Bedeutung sowie die sehr variable Zahl, Art und Lokalisation der Mutationen im jeweiligen Gen(abschnitt) erklären, zusammen mit dem Einfluss epigenetischer Faktoren, das breite geno- und phänotypische Spektrum der EB, das klinisch von milden (Blasenbildung bei längerem Gehen) bis zu schweren, mutilierenden und auch tödlich verlaufenden Formen reicht.

Die prognostisch bedeutende Unterscheidung der EB-Haupttypen erfolgt anhand der Ebene der Spaltbildung.

\section{Klassifikation}

\section{$\nabla$}

Die Heterogenität des klinischen Bildes erfordert eine Klassifikation, um eine unter Zuhilfenahme feingeweblicher (Routinehistologie, Ultrastruktur, Immunhistochemie) und genetischer Kriterien (Mutationsanalyse) exakte Diagnose- und damit Prognosestellung zu erzielen. Das präzise Wissen um den spezifischen EB-Subtyp ist die Voraussetzung für eine individuelle Festlegung von therapeutischen Optionen sowie die genetische Beratung der Familien. Die in den letzten Jahren erzielten Fortschritte im Verständnis der Pathogenese der EB und in der systematischen

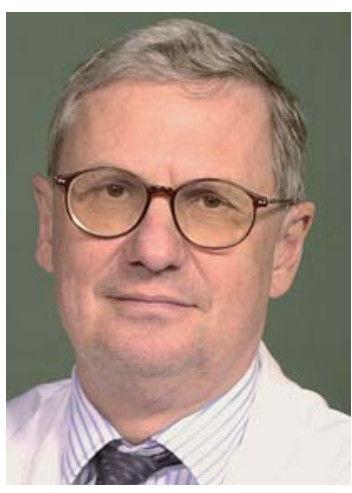

Prof. Dr. Helmut Hintner

VNR

2760512010047430456

Bibliografie

Dol http://dx.doi.org/

10.1055/s-2008-1077743

Online-Publikation: 19.5.2009

Akt Dermatol 2010; 36:

43-59 @ Georg Thieme

Verlag KG Stuttgart · New York ISSN 0340-2541

Korrespondenzadresse

Prof. Dr. Helmut Hintner

Universitätsklinik

für Dermatologie

Paracelsus Medizinische Privat-

universität (PMU) Salzburg

Salzburger Landeskliniken

(SALK)

Müllner Hauptstraße 48

A-5020 Salzburg

Österreich

h.hintner@salk.at 
Abb. 1 Oben: Schema der dermo-epidermalen Basalmembranzone (BMZ) mit Lokalisation der bei EB pathologisch veränderten Strukturproteine $(\mathrm{K} 5=$ Keratin $5, \mathrm{~K} 14=$ Keratin 14; EBS = Epidermolysis bullosa simplex, EB] $=$ Epidermolysis bullosa junctionalis, EBD = Epidermolysis bullosa dystrophicans). Unten: elektronenmikroskopische Darstellung der BMZ ( $\mathrm{KF}=$ Keratinfilamente, $\mathrm{LL}=$ Lamina lucida, $\mathrm{LD}=$ Lamina densa, $\mathrm{AF}=$ Ankerfibrillen).

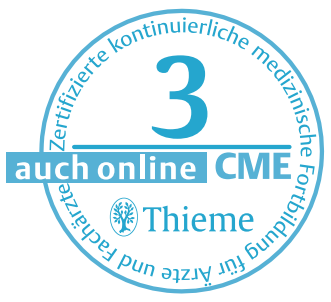

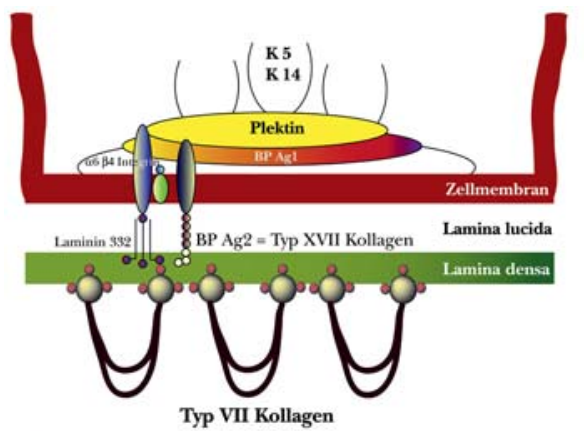

EBS

EBJ

EBD

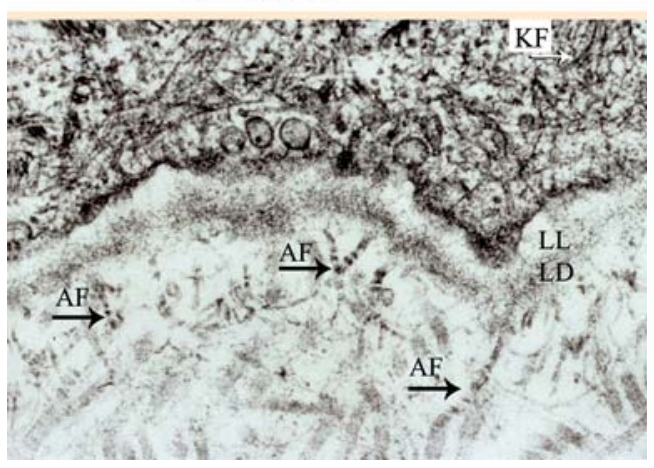

Charakterisierung eines immer umfassenderen Patientenkollektivs (das US National EB Registry umfasst Daten von 3280 EB-PatientInnen) flossen in die im Rahmen einer Konsensuskonferenz in Wien 2007 überarbeitete, neue Klassifikation der EB ein [1]. Nach dieser werden nun in Anlehnung an die ultrastrukturelle Ebene der Spaltbildung 4 Haupttypen unterschieden:

- die intraepidermale EB simplex (EBS),

- die junktionale EB (EBJ),

- die dermolytische EB (EBD) und

- Mischtypen wie das Kindler-Syndrom.

Weiterhin kommt es zur Unterteilung dieser Hauptgruppen in diverse Untergruppen, die in - Tab. 1 angeführt sind.

Im Folgenden werden die Hauptgruppen kurz beschrieben, wobei in Bezug auf Details auf das Buch „Life with epidermolysis bullosa: Etiology, diagnosis, interdisciplinary care and therapy“ verwiesen wird [2].

\section{Epidermolysis bullosa simplex (EBS)}

Man unterscheidet lokale und generalisierte Formen der meist autosomal dominant vererbten EBS. Die lokale EBS ist weithin am häufigsten und die pathognomonische Blasen- und Erosionsbildung meist auf die mechanisch besonders exponierte Hand- und Fußregion begrenzt. Mit Ausnahme diskreter Blasen in der Mundschleimhaut sind Extrakutanmanifestationen sehr selten.

Die bedeutendsten generalisierten Formen, die EBS-Dowling Meara (EBS-DM) und EBS-nonDowling Meara (EBS, gen-nonDM), zeigen neben den am gesamten Integument auftretenden, bei der EBS-DM typischerweise herpetiform gruppierten Blasen und Erosionen ( $\bullet$ Abb.2) häufig ausgeprägte palmoplantare Hyperkeratosen, $\mathrm{Na}$ -

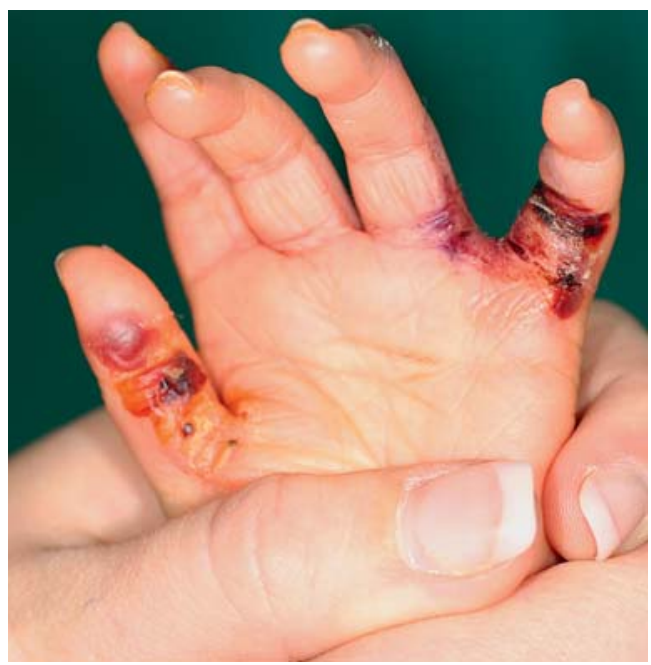

Abb. 2 Herpetiform gruppierte, teils hämorrhagische Blasen, Erosionen und Krusten bei EBS-DM.

geldystrophie, atrophe Narben, Milien und regulär einen Schleimhautbefall.

Die EBS-DM kann zudem durch Wachstumsverzögerung und Larynxstenosen kompliziert sein und weist eine erhöhte Mortalität auf.

Molekularpathologisch liegen den meisten EBSVarianten Mutationen in den für Keratin 5 oder 14 kodierenden Genen zugrunde. Je nach der Qualität der Mutation (Lokalisation, Homo- oder Heterozygotie) kommt es zur vollständigen (und damit z. B. bei der EBS-DM phänotypisch schwerwiegenderen) oder nur teilweisen (bei der lokalen EBS klinisch leichter verlaufenden) Hemmung der Aggregation von Keratinfilamenten (Tonofilamente) in den basalen Keratinozyten. Die damit verbundene Destabilisierung des Zytoskeletts verursacht schließlich eine Zytolyse. Das hemidesmosomale Strukturprotein Plektin, das auch in den „z-disks“ von Muskelzellen vorkommt, ist bei der seltenen Variante EBS mit Muskeldystrophie (EBS-MD) durch eine Mutation im entsprechenden Gen verändert, was neben den mit der Geburt auftretenden typischen Hautund Schleimhautläsionen zu einer sich erst zwischen dem 2. und 35. Lebensjahr verzögert manifestierenden, progredienten Muskeldystrophie führt.

Mutationen in den für Keratin 5 oder 14 kodierenden Genen liegen der EB simplex zugrunde. Sie verursachen eine Zytolyse von Keratinozyten mit folgender intraepidermaler Spaltbildung. Die kutanen Läsionen können lokal oder generalisiert auftreten.

\section{Epidermolysis bullosa junctionalis (EBJ)}

EBJ-Herlitz. Bei der EBJ werden 2 wichtige, autosomal rezessiv vererbte Subtypen beschrieben. Die schwere und häufig tödlich verlaufende EBJ- 
Tab. 1 Klinik und Pathologie der EB hereditaria Haupt- und Untergruppen (nach [1]).

\begin{tabular}{|c|c|c|c|c|}
\hline Hauptgruppe & Häufigste Subypen & Vererbung & $\begin{array}{l}\text { Mutationen nach- } \\
\text { gewiesen im Gen für }\end{array}$ & Klinik \\
\hline \multirow{14}{*}{$\begin{array}{l}\text { EB simplex (EBS) } \\
\text { intraepidermale = } \\
\text { epidermolytische } \\
\text { Spaltbildung }\end{array}$} & \multicolumn{4}{|c|}{ Suprabasale EBS (Blasenbildung durch Zytolyse suprabasaler Keratinozyten) } \\
\hline & letale acantholytische EBS & $\mathrm{AR}$ & Desmoplakin & $\begin{array}{l}\text { mit Geburt generalisiert oberflächliche Ero- } \\
\text { sionen, aber keine Blasen, auch in Oropharynx, } \\
\text { Gastrointestinal-, Urogenital- und Respira- } \\
\text { tionstrakt; Alopezie, Nageldystrophie und } \\
\text {-verlust; frühe postpartale Letalität }\end{array}$ \\
\hline & EBS mit Plakophilin-Defizienz & AR & Plakophilin-1 & $\begin{array}{l}\text { mit Geburt generalisiert oberflächliche Erosio- } \\
\text { nen, selten Blasen; Nageldystrophie und -ver- } \\
\text { lust, fokal Keratoderm, Hypotrichose, periorale } \\
\text { und Zungen-Fissuren; Wachstumsretardierung, } \\
\text { Obstipation, Ösophagusstrikturen, Blepharitis, } \\
\text { verminderte oder fehlende Wimpern }\end{array}$ \\
\hline & $\begin{array}{l}\text { EBS superficialis } \\
\text { (EBSS) } \\
\text { Spaltbildung zwischen Stra- } \\
\text { tum granulosum und corneum }\end{array}$ & $A D$ & $?$ & $\begin{array}{l}\text { mit Geburt oder in früher Kindheit generalisiert } \\
\text { oder akral oberflächliche Erosionen, keine Bla- } \\
\text { sen; Milien, atrophe Narben, Nageldystrophie }\end{array}$ \\
\hline & \multicolumn{4}{|c|}{ Basale EBS (Blasenbildung durch Zytolyse basaler Keratinozyten) } \\
\hline & $\begin{array}{l}\text { lokale EBS } \\
\text { (EBS-loc) } \\
\text { vormals EBS-Weber-Cockayne }\end{array}$ & $A D$ & Keratin 5,14 & $\begin{array}{l}\text { in früher Kindheit Blasen, Erosionen, Krusten } \\
\text { palmar und plantar, vorwiegend im Sommer; } \\
\text { Erosionen der Mundschleimhaut in } 25 \%\end{array}$ \\
\hline & $\begin{array}{l}\text { generalisierte EBS, } \\
\text { andere/non-DM } \\
\text { (EBS, gen-nonDM) } \\
\text { vormals EBS-Köbner }\end{array}$ & $\begin{array}{l}A D \text {, selten } \\
A R\end{array}$ & $\begin{array}{l}\text { Keratin } 5,14 \text {; intra- } \\
\text { zelluläre Domäne von } \\
\text { Integrin } \beta 4\end{array}$ & $\begin{array}{l}\text { von Geburt an generalisiert Blasen, Erosionen, } \\
\text { Krusten mit Betonung der Extremitäten; Milien, } \\
\text { atrophe Narben, Nageldystrophie, -verlust, } \\
\text { fokal palmoplantares Keratoderm; Mitbeteili- } \\
\text { gung der Mundschleimhaut, selten Augen- } \\
\text { beteiligung }\end{array}$ \\
\hline & $\begin{array}{l}\text { EBS-Dowling-Meara } \\
\text { (EBS-DM) }\end{array}$ & $A D$ & Keratin 5,14 & $\begin{array}{l}\text { mit Geburt generalisiert Blasen (in herpeti- } \\
\text { former Anordnung), Erosionen, Krusten unter } \\
\text { Mitbeteiligung der Mundschleimhaut; palmo- } \\
\text { plantare Hyperkeratosen, Nageldystrophien, } \\
\text { atrophe Narbenbildungen, Milien; Anämie, } \\
\text { Wachstumsretardierung, Obstipation }\end{array}$ \\
\hline & $\begin{array}{l}\text { EBS-Muskeldystrophie } \\
\text { (EBS-MD) }\end{array}$ & $A R,(A D)$ & $\begin{array}{l}\text { Plektin (fehlend oder } \\
\text { vermindert) }\end{array}$ & $\begin{array}{l}\text { mit Geburt generalisiert Blasen, Erosionen, } \\
\text { Krusten, Milien, atrophe Narben, Nageldystro- } \\
\text { phie und -verlust; stenosierende Granulationen } \\
\text { im Respirationstrakt; progrediente Muskeldys- } \\
\text { trophie zw. 2.- } 35 \text {. Lebensjahr; erhöhte Letalität }\end{array}$ \\
\hline & $\begin{array}{l}\text { EBS mit Pylorusatresie } \\
\text { (EBS-PA) }\end{array}$ & $?$ & $\begin{array}{l}\text { Plektin (fehlend oder } \\
\text { vermindert), Integrin } \\
\alpha 6 \beta 4 \text { (fehlend oder } \\
\text { vermindert) }\end{array}$ & $\begin{array}{l}\text { mit Geburt generalisiert Blasen, Erosionen, } \\
\text { teilweise großflächige kongenitale Haut- } \\
\text { defekte; atrophe Narben; Mundschleimhaut- } \\
\text { beteiligung, Deformationen der Ohrmuscheln } \\
\text { und Alae nasi; Pylorusatresie; Anämie, Wachs- } \\
\text { tumsretardierung, Gelenkskontrakturen, } \\
\text { Kryptorchismus; hohe Letalität }\end{array}$ \\
\hline & $\begin{array}{l}\text { EBS, autosomal rezessiv } \\
\text { (EBS-AR) }\end{array}$ & AR & Keratin 14 & $\begin{array}{l}\text { mit Geburt generalisiert Blasen (besonders } \\
\text { anogenital), selten Milien oder atrophe Narben; } \\
\text { Nageldystrophie, ichthyosiforme Plaques; } \\
\text { Mundschleimhautbeteiligung, Karies; } \\
\text { Extrakutanmanifestationen: Anämie, Wachs- } \\
\text { tumsretardierung, Obstipation, Urogenital- } \\
\text { traktbeteiligung }\end{array}$ \\
\hline & $\begin{array}{l}\text { EBS mit scheckiger (mottled) } \\
\text { Pigmentierung } \\
\text { (EBS-MP) }\end{array}$ & $\mathrm{AD}$ & Keratin 5 & $\begin{array}{l}\text { mit Geburt generalisiert Blasenbildung, } \\
\text { fleckige oder retikuläre braune Pigmentierung }\end{array}$ \\
\hline & $\begin{array}{l}\text { EBS Ogna } \\
\text { (EBS-Og) }\end{array}$ & $\mathrm{AD}$ & Plektin (vermindert) & $\begin{array}{l}\text { mit Geburt vorrangig akrale Blasenbildung, } \\
\text { Onychogryphose }\end{array}$ \\
\hline & $\begin{array}{l}\text { EBS, zirzinär-wandernd } \\
\text { (migratory circinate) } \\
\text { (EBS-migr) }\end{array}$ & $A D$ & $\begin{array}{l}\text { Keratin } 5 \text { (fehlend } \\
\text { oder vermindert) }\end{array}$ & $\begin{array}{l}\text { mit Geburt generalisiert Blasenbildung, } \\
\text { migratorisches circinäres Erythem, braune } \\
\text { postinflammatorische Hyperpigmentierung }\end{array}$ \\
\hline
\end{tabular}




\begin{tabular}{|c|c|c|c|c|}
\hline Hauptgruppe & Häufigste Subypen & Vererbung & $\begin{array}{l}\text { Mutationen nach- } \\
\text { gewiesen im Gen für }\end{array}$ & Klinik \\
\hline \multirow{9}{*}{$\begin{array}{l}\text { EB junctionalis } \\
\text { (EBJ) } \\
\text { junktionale } \\
\text { Spaltbildung in } \\
\text { der Lamina lucida } \\
\text { der dermo-epider- } \\
\text { malen BMZ }\end{array}$} & EBJ-Herlitz (JEB-H) & & & \\
\hline & $\begin{array}{l}\text { EBJ-Herlitz } \\
(\text { JEB-H) }\end{array}$ & AR & $\begin{array}{l}\text { Laminin } 332 \text { (fehlend } \\
\text { oder vermindert) }\end{array}$ & $\begin{array}{l}\text { mit Geburt generalisiert Blasen- und atrophe } \\
\text { Narbenbildung, chronisch-granulomatöse } \\
\text { Erosionen, Onychodystrophie und Nagel- } \\
\text { verlust, narbige Alopezie, Milien; Pseudosyn- } \\
\text { daktylie; Mundschleimhautbeteiligung, Mikro- } \\
\text { stomie, Ankyloglossie, Zahnschmelzhypo- } \\
\text { plasie, exzessive Karies; Extrakutanmanifesta- } \\
\text { tionen: Anämie, Wachstumsretardierung, } \\
\text { Beteiligung des Gastrointestinal-, Respirations- } \\
\text { und Urogenitaltrakts, Beteiligung der Konjunk- } \\
\text { tiven; verzögerte Pubertät, hohe Letalität }\end{array}$ \\
\hline & EBJ, andere (JEB-O) & & & \\
\hline & $\begin{array}{l}\text { generalisierte EBJ-non-Herlitz } \\
\text { (JEB-nH, gen) } \\
\text { Vormals Generalisierte atrophe } \\
\text { benigne EB (GABEB) }\end{array}$ & AR & $\begin{array}{l}\text { Laminin } 332 \\
\text { (vermindert), } \\
\text { Typ-XVII-Kollagen = } \\
\text { bullöses-Pemphigoid- } \\
\text { Antigen-2 (BPAG-2) } \\
\text { (fehlend) }\end{array}$ & $\begin{array}{l}\text { mit Geburt generalisiert Blasen, Erosionen, } \\
\text { Krusten, atrophe Narben, Milien, „male-pat- } \\
\text { tern“-Alopezie, Nageldystrophie bzw. -verlust; } \\
\text { Mundschleimhautbeteiligung; exzessive Karies } \\
\text { und Zahnverlust, Zahnschmelzhypoplasie; } \\
\text { Extrakutanmanifestationen: Anämie, Wachs- } \\
\text { tumsretardierung, Beteiligung von Gastro- } \\
\text { intestinal-, Respirations- und Urogenitaltrakt } \\
\text { sowie der Konjunktiven }\end{array}$ \\
\hline & $\begin{array}{l}\text { Lokale EBJ-non-Herlitz, } \\
\text { (JEB-nH, loc) }\end{array}$ & AR & $\begin{array}{l}\text { Bullöses-Pemphigoid- } \\
\text { Antigen-2 (BPAG-2 = } \\
\text { Typ-XVII-Kollagen) } \\
\text { (vermindert) }\end{array}$ & $\begin{array}{l}\text { mit Geburt lokales Auftreten von Blasen, } \\
\text { Milien, Nageldystrophie und -verlust, Mitbefall } \\
\text { von Mundschleimhaut, Zahnschmelzhypo- } \\
\text { plasie, exzessive Karies }\end{array}$ \\
\hline & $\begin{array}{l}\text { EBJ mit Pylorusatresie } \\
\text { (JEB-PA) }\end{array}$ & AR & $\begin{array}{l}\text { Integrin } \alpha 6 \text { und } \beta 4 \\
\text { (fehlend oder } \\
\text { vermindert) }\end{array}$ & $\begin{array}{l}\text { mit Geburt generalisiert massive Blasenbil- } \\
\text { dung, Erosionen, Krusten; atrophe Narben, } \\
\text { Nageldystrophie und -verlust, tw. Assoziation } \\
\text { mit großflächig-kongenitaler Aplasia cutis, } \\
\text { Zahnschmelzhypoplasie; Pylorusatresie; kon- } \\
\text { genitale urogenitale Malformationen; verein- } \\
\text { zelt rudimentäre Ohrenanlage; hohe Letalität }\end{array}$ \\
\hline & $\begin{array}{l}\text { JEB inversa } \\
\text { (JEB-I) }\end{array}$ & AR & Laminin 332 & $\begin{array}{l}\text { mit Geburt in intertriginösen Arealen Blasen, } \\
\text { atrophe Narben, Nageldystrophie, Karies, } \\
\text { Extrakutanmanifestationen: Gastrointestinal- } \\
\text { traktbeteiligung }\end{array}$ \\
\hline & $\begin{array}{l}\text { JEB, später Beginn (late onset) } \\
\text { JEB-lo) }\end{array}$ & AR & $?$ & $\begin{array}{l}\text { Blasenbildung in Adoleszenz oder später, } \\
\text { Nageldystrophie und -verlust, Hyperhidrose, } \\
\text { fehlende Dermatoglyphen, Zahnschmelz- } \\
\text { hypoplasie }\end{array}$ \\
\hline & $\begin{array}{l}\text { laryngo-onychokutanes } \\
\text { Syndrom Shabbir }\end{array}$ & AR & Laminin $332 \alpha 3$ Kette & $\begin{array}{l}\text { mit Geburt Erosionen im Respirationstrakt } \\
\text { (Larynx), an Haut vorrangig im Gesicht und } \\
\text { nuchal, Nageldystrophie und -verlust, granu- } \\
\text { lierende Entzündung mit atropher Narben- } \\
\text { bildung; erhöhte Inzidenz in Punjab, Zahn- } \\
\text { schmelzhypoplasie, Karies; Mitbeteiligung der } \\
\text { Konjunktiven }\end{array}$ \\
\hline
\end{tabular}




\begin{tabular}{|c|c|c|c|c|}
\hline Hauptgruppe & Häufigste Subypen & Vererbung & $\begin{array}{l}\text { Mutationen nach- } \\
\text { gewiesen im Gen für }\end{array}$ & Klinik \\
\hline \multirow{11}{*}{$\begin{array}{l}\text { EB dystrophicans } \\
\text { (EBD) } \\
\text { "Dermolytische“ } \\
\text { Spaltbildung unter- } \\
\text { halb der Lamina } \\
\text { densa der dermo- } \\
\text { epidermalen BMZ }\end{array}$} & Dominante EBD & & & \\
\hline & $\begin{array}{l}\text { generalisierte dominante EBD } \\
\text { (DDEB-gen) }\end{array}$ & $A D$ & Typ-VII-Kollagen & $\begin{array}{l}\text { mit Geburt generalisiert Blasenbildung, Ero- } \\
\text { sionen, Milien, atrophe Narben, Pseudosyndak- } \\
\text { tylie, Nageldystrophie und -verlust, Alopezie, } \\
\text { „albopapuloide Läsionen“ (gruppierte, hypo- } \\
\text { pigmentierte Papeln), symptomatische Beteili- } \\
\text { gung der Mundschleimhaut und des Gastro- } \\
\text { intestinal- und selten des Urogenitaltraktes }\end{array}$ \\
\hline & $\begin{array}{l}\text { akrale DEBD } \\
\text { (DDEB-ac) }\end{array}$ & $A D, A R$ & Typ-VII-Kollagen & $\begin{array}{l}\text { mit früher Kindheit an Händen und Füßen } \\
\text { Blasen, Erosionen, Milien, atrophe Narben, } \\
\text { Nageldystrophie }\end{array}$ \\
\hline & $\begin{array}{l}\text { prätibiale DEBD } \\
\text { (DDEB-Pt) }\end{array}$ & $A D, A R$ & Typ-VII-Kollagen & $\begin{array}{l}\text { mit Geburt oder früher Kindheit Blasen, Ero- } \\
\text { sionen prätibial, an Händen und Füßen; Milien, } \\
\text { atrophe Narben, Nageldystrophie, Lichen } \\
\text { planus-artige Hautläsionen, exzessive Karies, } \\
\text { Obstipation }\end{array}$ \\
\hline & $\begin{array}{l}\text { pruriginöse DEBD } \\
\text { (DDEB-Pr) }\end{array}$ & $A D, A R$ & Typ-VII-Kollagen & $\begin{array}{l}\text { mit Kindheit generalisiert oder lokalisiert } \\
\text { Erosionen und Blasen, atrophe Narben, Milien, } \\
\text { Nageldystrophie; ausgeprägter Juckreiz, Ob- } \\
\text { stipation; möglicherweise erhöhtes Risiko für } \\
\text { Entwicklung von Plattenepithelkarzinomen ab } \\
\text { mittlerem Lebensalter }\end{array}$ \\
\hline & $\begin{array}{l}\text { DEBD mit Nageldystrophie } \\
\text { (DDEB-na) }\end{array}$ & $A D$ & Typ-VII-Kollagen & $\begin{array}{l}\text { mit Geburt oder in Kindheit Nageldystrophie } \\
\text { und -verlust }\end{array}$ \\
\hline & $\begin{array}{l}\text { DEBD, bullous dermolysis of } \\
\text { the newborn } \\
\text { (DDEB-BDN) }\end{array}$ & $A D, A R$ & $\begin{array}{l}\text { Typ-VII-Kollagen } \\
\text { (granuläres Färbe- } \\
\text { profil in basalen und } \\
\text { suprabasalen Kerati- } \\
\text { nozyten; fehlend oder } \\
\text { vermindert entlang } \\
\text { der dermo-epider- } \\
\text { malen Junktionszone) }\end{array}$ & $\begin{array}{l}\text { mit Geburt oder früher Kindheit generalisiert } \\
\text { Blasen, Erosionen, Milien, atrophe Narben, } \\
\text { Nageldystrophie; exzessive Karies }\end{array}$ \\
\hline & Rezessive EBD & & & \\
\hline & $\begin{array}{l}\text { schwere, generalisierte REBD } \\
\text { (RDEB-sev gen) } \\
\text { Vormals rezessive EBD-Hallo- } \\
\text { peau-Siemens }\end{array}$ & $\mathrm{AR}$ & $\begin{array}{l}\text { Typ-VII-Kollagen } \\
\text { (fehlend oder } \\
\text { vermindert) }\end{array}$ & $\begin{array}{l}\text { mit Geburt generalisiert Blasen, Erosionen, } \\
\text { Krusten; atrophe Narben, Milien, narbige Alo- } \\
\text { pezie, Nagelverlust, Mutilationen an Händen } \\
\text { und Füßen, Pseudosyndaktylien, Kontrakturen; } \\
\text { Mundschleimhautbeteiligung, exzessive } \\
\text { Karies; multiple Angiome, Extrakutanmanifes- } \\
\text { tationen: ausgeprägte Wachstumsretardation, } \\
\text { Anämie, Konjunktiven- und Gastrointestinal- } \\
\text { trakt-Beteiligung, Glomerulonephritiden, } \\
\text { renale Amyloidose, IgA- Nephropathie, chro- } \\
\text { nische Niereninsuffizienz, Kardiomyopathie, } \\
\text { Osteoporose, Plattenepithelkarzinome; ver- } \\
\text { zögerte Pubertät, hohe Letalität }\end{array}$ \\
\hline & $\begin{array}{l}\text { generalisierte REBD, andere } \\
\text { (RDEB-O) } \\
\text { Vormals REBD-non-Hallopeau- } \\
\text { Siemens } \\
\text { (REBD-nHS) }\end{array}$ & $\mathrm{AR}$ & $\begin{array}{l}\text { Typ-VII-Kollagen } \\
\text { (vermindert) }\end{array}$ & $\begin{array}{l}\text { mit Geburt generalisiert Blasen, Erosionen, } \\
\text { Krusten; atrophe Narben, Milien, Nageldystro- } \\
\text { phie, narbige Alopezie; Pseudosyndaktylie, } \\
\text { Plattenepithelkarzinome; Mundschleimhaut- } \\
\text { beteiligung } \\
\text { Extrakutanmanifestationen: Wachstums- } \\
\text { retardation, Anämie, konjunktivale und } \\
\text { Gastrointestinal- und Urogenitaltraktbeteili- } \\
\text { gung; erhöhte Letalität }\end{array}$ \\
\hline & $\begin{array}{l}\text { REBD inversa } \\
\text { (RDEB-I) }\end{array}$ & $\mathrm{AR}$ & $\begin{array}{l}\text { Typ-VII-Kollagen } \\
\text { (variabel exprimiert) }\end{array}$ & $\begin{array}{l}\text { mit Geburt in intertriginösen Arealen, lumbo- } \\
\text { sakral, akral und axial Blasen, Milien, atrophe } \\
\text { Narben, Nageldystrophie } \\
\text { Extrakutanmanifestationen: Anämie, Wachs- } \\
\text { tumsretardierung, Beteiligung von Mund- } \\
\text { schleimhaut, Gastrointestinal- und Urogenital- } \\
\text { trakt, erhöhte Kariesrate; Stenose des äußeren } \\
\text { Gehörganges }\end{array}$ \\
\hline
\end{tabular}




\begin{tabular}{|c|c|c|c|c|}
\hline Hauptgruppe & Häufigste Subypen & Vererbung & $\begin{array}{l}\text { Mutationen nach- } \\
\text { gewiesen im Gen für }\end{array}$ & Klinik \\
\hline \multirow{5}{*}{$\begin{array}{l}\text { EB dystrophicans } \\
\text { (EBD) } \\
\text { „Dermolytische“ } \\
\text { Spaltbildung unter- } \\
\text { halb der Lamina } \\
\text { densa der dermo- } \\
\text { epidermalen BMZ }\end{array}$} & $\begin{array}{l}\text { prätibilae REBD } \\
\text { (RDEB-Pt) }\end{array}$ & $A D, A R$ & Typ-VII-Kollagen & $\begin{array}{l}\text { mit Geburt oder früher Kindheit Blasen, Erosio- } \\
\text { nen prätibial, an Händen, Füßen und Nägeln; } \\
\text { Milien, atrophe Narben, Nageldystrophie, } \\
\text { Lichen planus-artige Hautläsionen; exzessive } \\
\text { Karies, Obstipation }\end{array}$ \\
\hline & $\begin{array}{l}\text { akrale REBD } \\
\text { (RDEB-ac) }\end{array}$ & $A D, A R$ & Typ-VII-Kollagen & $\begin{array}{l}\text { mit früher Kindheit an Händen und Füßen } \\
\text { Blasen, Erosionen, Milien, atrophe Narben, } \\
\text { Nageldystrophie }\end{array}$ \\
\hline & $\begin{array}{l}\text { RDEB pruriginosa } \\
\text { (RDEB-Pr) }\end{array}$ & AR & Typ-VII-Kollagen & $\begin{array}{l}\text { Erosionen und Blasen, atrophe Narben, Milien, } \\
\text { Nageldystrophie; ausgeprägter Juckreiz }\end{array}$ \\
\hline & $\begin{array}{l}\text { RDEB centripetalis } \\
\text { (RDEB-Ce) }\end{array}$ & $A R$ & Typ-VII-Kollagen & $\begin{array}{l}\text { mit Geburt oder in früher Kindheit prätibial und } \\
\text { akral Blasen, Milien, atrophe Narben, Nagel- } \\
\text { dystrophie } \\
\text { Extrakutanmanifestationen: Mundschleim- } \\
\text { hautbeteiligung }\end{array}$ \\
\hline & $\begin{array}{l}\text { RDEB, bullous dermolysis } \\
\text { of the newborn } \\
\text { (RDEB-BDN) }\end{array}$ & AR & Typ-VII-Kollagen & \\
\hline $\begin{array}{l}\text { Kindler Syndrom } \\
\text { Intraepidermale, } \\
\text { junktionale oder } \\
\text { sublamina densa } \\
\text { Spaltbildung }\end{array}$ & & $A R$ & $\begin{array}{l}\text { Kindlin-1 (inter- } \\
\text { zelluläres Adhäsions- } \\
\text { protein basaler Kera- } \\
\text { tinozyten) (fehlend, } \\
\text { vermindert oder } \\
\text { normal bei homozy- } \\
\text { goter loss-of-function } \\
\text { Mutation in KIND1) }\end{array}$ & $\begin{array}{l}\text { mit Geburt generalisiert Blasen, Erosionen, } \\
\text { Krusten, atrophe Narben, Poikilodermie; Pho- } \\
\text { tosensitivität, Hautfragiliät nimmt mit Alter ab; } \\
\text { Extrakutanmanifestationen: Gingivahyper- } \\
\text { plasie, Kolitis, Ösophagitis, Genitaltraktbetei- } \\
\text { ligung, mentale Retardierung, Ektropion, } \\
\text { Knochenmissbildungen }\end{array}$ \\
\hline
\end{tabular}

$A R=$ autosomal rezessiv, $A D=$ autosomal dominant.

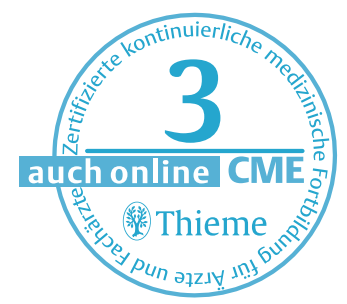

Herlitz ist auf homozygote Nonsense-Mutationen in einem der für die Ketten LAMA3, LAMB3 und LAMC2 des Heterotrimers Laminin 332 kodierenden Gene zurückzuführen. Der mit dieser Mutation verbundene Abbau der messenger-RNA bedingt den vollständigen, bi-allelischen Verlust dieses BMZ-Strukturproteins. Damit verbunden sind eine ausgeprägte Blasenbildung, großflächige Erosionen, atrophe Narben, Nageldystrophie bzw. -verlust, Milien, die Bildung exzessiven Granulationsgewebes periorifiziell im Gesicht sowie eine schwere Mundschleimhautbeteiligung ( Abb. 3).

Ausgeprägte Karies, multifaktorielle Anämie, Wachstumsretardierung, Mitbeteiligung von Respirations- und Urogenitaltrakt wie auch der konjunktivalen Schleimhaut sind Zeichen einer Systembeteiligung, die durch Sepsis, Pneumonien oder laryngotracheale Obstruktion häufig in den ersten beiden Lebensjahren zum Tode führt. EBJ-non-Herlitz. Die generalisierte EBJ-non-Herlitz (EBJ-nH) wird entweder durch kombiniertheterozygote Mutationen der Laminin-332 Ketten-Gene oder durch homozygote nonsense und missense Mutationen bzw. inframe Deletionen im für das Typ-XVII-Kollagen (Strukturprotein der Ankerfilamente) kodierenden COL17A1-Gen verursacht. Die damit zum Teil erhalten bleibende Funktion des Genproduktes erlaubt einen etwas milderen Phänotyp. EBJ-nH-Patienten leiden dennoch unter einer generalisierten Blasenbildung mit Erosionen, Krusten, atrophen Narben, Nageldystrophie und -verlust, Zahnschmelzhypoplasie, Karies und vernarbender („male pat- tern“) Alopezie. Extrakutanmanifestationen sind aber, im Gegensatz zur EBJ-H, mit Ausnahme von laryngotrachealen Stenosen selten und die Lebenserwartung der Betroffen ist nicht maßgeblich eingeschränkt.

Pylorusatresie. Eine seltene Variante der EBJ stellt der Subtyp mit Pylorusatresie (EBJ-PA) dar. Der genetisch bedingte Verlust von funktionalem a6ß4-Integrin in Hemidesmosomen von Epithelzellen, Schwann'schen Zellen, Thymozyten und neuronalen Fibroblasten resultiert in einem oft durch zusätzlich auftretende Fehlbildungen im Urogenitaltrakt komplizierten, schweren Verlauf der EB, der meist frühzeitig tödlich endet. Vor Kurzem wurde auch eine nicht-letale, mildere Variante auf Basis einer compound-heterozygoten Mutation beschrieben [3]. Ein klinisch korrespondierender Subtyp besteht auch als Variante der EBS [1].

Die EB junctionalis mit Spaltbildung innerhalb der Lamina lucida wird durch ein defektes Laminin 332, $\alpha 6 \beta 4$-Integrin oder Typ-XVII-Kollagen verursacht. Homozygote Nonsense-Mutationen führen zur schweren, meist fatalen Verlaufsform EBJ-Herlitz mit zahlreichen Extrakutanmanifestationen, kombiniert heterozygote Genveränderungen zur milderen Variante EBJ-non-Herlitz. 


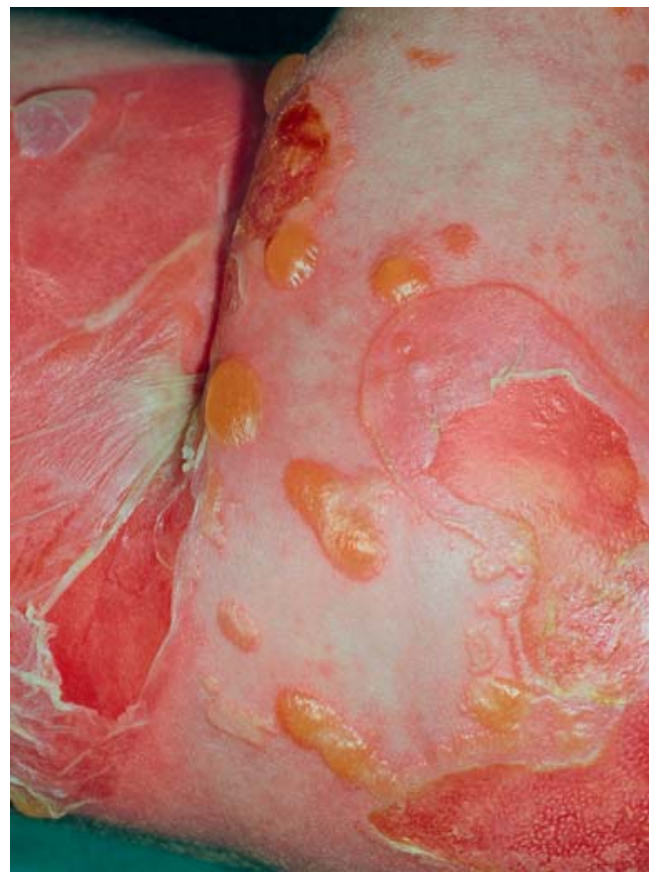

Abb. 3 EBJ-Herlitz. Generalisierte Blasen- und Erosionsbildung sowie großflächiges Abschwimmen der Epidermis.

Epidermolysis bullosa dystrophicans (EBD) Ursache für die EBD sind Mutationen in dem für Typ-VII-Kollagen kodierenden Gen COL7A1. TypVII-Kollagen stellt die Hauptstrukturkomponente der Ankerfibrillen dar, sein Verlust führt zur charakteristischen dermolytischen Spaltbildung unter der Lamina densa der dermo-epidermalen BMZ. Eine Vielzahl an meist familienspezifischen Mutationen wurde beschrieben.

Die EBD kann, basierend auf ihrem Vererbungsmuster, in 2 Subtypen unterteilt werden. Bei der autosomal dominant vererbten EBD (DEBD) kommt es neben dem zumeist generalisierten Auftreten von Blasen, Erosionen, Milien sowie atropher Vernarbung ( $\bullet$ Abb.4) zusätzlich zu Nageldystrophie und -verlust und Manifestationen im Ösophagus, während andere Extrakutansymptome nahezu immer fehlen.

Das molekulare Substrat sind hierbei MissenseMutationen im Gen für die pro- $\alpha$-Kette des Homotrimers Typ-VII-Kollagen, die zur Destabilisierung der Tripelhelix führen. Da sowohl das defekte als auch das korrekte Allel gleichwertig exprimiert werden, sind 12,5\% der Ankerfibrillen funktionell intakt, was den relativ milden Verlauf der dominanten EBD-Variante erklärt.

Missense-Mutationen oder inframe Deletionen, die durch Interferenz mit der Aggregation von Typ-VII-Kollagen-Polypeptiden zur Bildung von in Zahl oder Morphologie veränderten Ankerfibrillen führen, unterliegen den milderen Formen der rezessiven EBD (REBD). Homozygote Premature Termination Codons (PTC) mit in der Folge komplettem Verlust von Typ-VII-Kollagen sind Ursache für die schwere, generalisierte autoso-

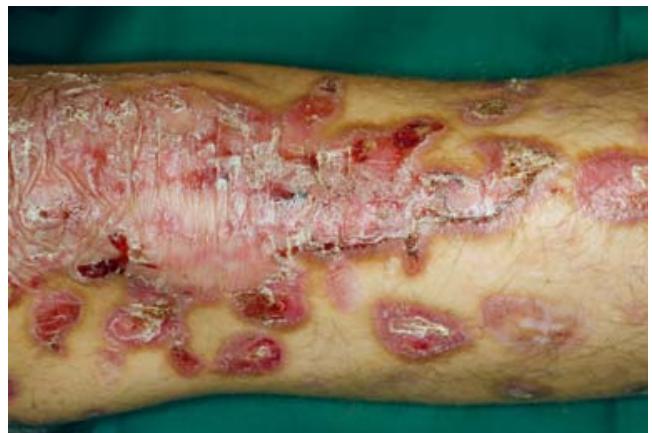

Abb. 4 Dominante EBD. Verkrustete Erosionen, Schuppen und atrophe, teils leicht hyperpigmentierte Narbenbildung im Bereich des Knies.

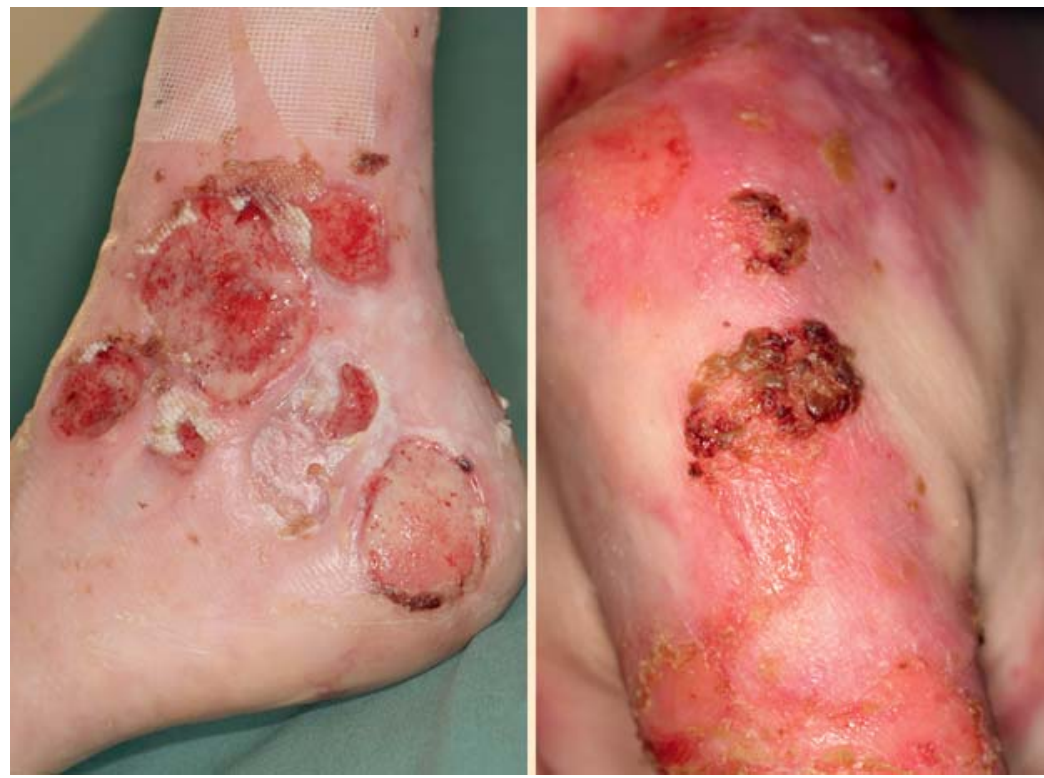

Abb. 5 Schlecht heilende, teils tiefreichende Ulzera (links) sowie superinfizierte Krusten und Erosionen (rechts) bei schwerer, generalisierter REBD.

mal rezessiv vererbte EBD (REBD) mit disseminierten Blasen, Erosionen, Krusten, atrophen Narben, Onychodystrophie und Nagelverlust, mutilierender Syndaktylie und Kontrakturen an Händen, Füßen, Ellbogen- sowie Kniegelenken (O Abb. 5).

Extrakutanmanifestationen sind bei dieser Variante häufig und betreffen vorrangig den Gastrointestinal- und Urogenitaltrakt sowie die Konjunktiven. Weiterhin sind Zahndefekte und -verlust, chronische Anämie, Wachstumsverzögerung und das enorm erhöhte Risiko für die frühzeitige Entwicklung von aggressiven und therapieresistenten Plattenepithelkarzinomen von klinischer Bedeutung.

Verändertes Typ-VII-Kollagen (Strukturprotein der Ankerfibrillen) führt zu dermolytischer Spaltbildung als Kennzeichen der EB dystrophicans. Es werden dominant und rezessiv vererbte Formen unterschieden. Atrophe Narben, Pseudosyndaktylien, Kontrakturen, aggressive Plattenepithelkarzinome sind häufig und verschiedenste Extrakutanmanifestationen meist schwerwiegend. 


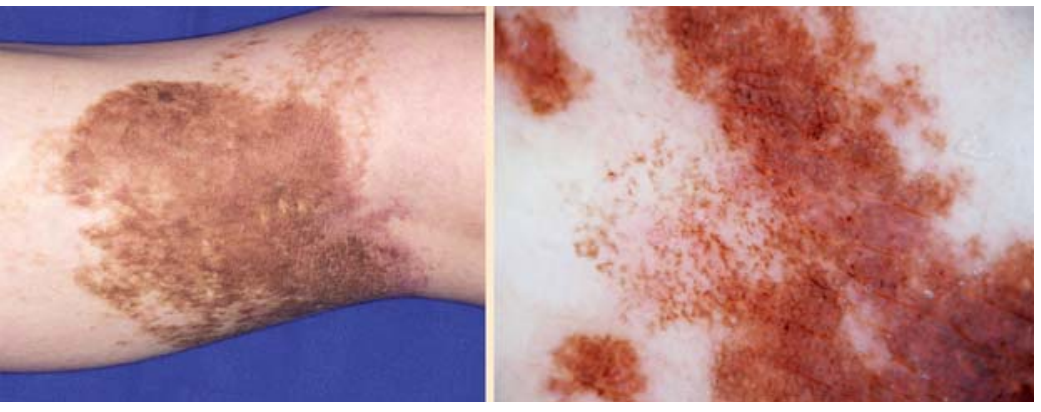

Abb. 6 Links: Handtellergroßer, asymmetrischer, irregulär pigmentierter EB-Nävus mit Ausläufern und Satellitenbildung in der Kniekehle eines Patienten mit generalisierter EBJ-non-Herlitz. Rechts: Dermatoskopische Aufnahme eines EB-Nävus bei EBS. Es zeigt sich ein irreguläres Pigmentnetzwerk, braune Schollen und Dots. Zentral überlagern zudem helle Areale mit $\mathrm{Hy}$ perkeratose.

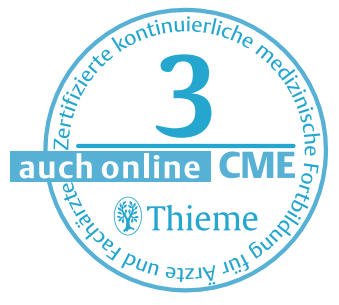

Kindler-Syndrom

Mit der neuesten Überarbeitung im Jahre 2007 wurde auch das Kindler-Syndrom (KS) als eigener 4. Subtyp in das Klassifikationssystem vererbter bullöser Epidermolysen aufgenommen (siehe $\square$ Tab. 1 für klinische Details). Die Rechtfertigung als eigene Entität, die sich typischerweise als vererbte mechanobullöse Hauterkrankung präsentiert, ergibt sich unter anderem aus dem Umstand, dass die Spaltbildung beim KS mehrere ultrastrukturelle Ebenen umfassen kann.

\section{Spezielle klinische Manifestationen $\nabla$}

\section{EB-Nävi}

EB-Nävi sind große, eruptive, asymmetrische, oft irregulär pigmentierte und hoch dynamische melanozytäre Läsionen mit scharf demarkierten Rändern, die typischerweise im Bereich vorangegangener Blasen bei vorrangig rezessiv vererbten Formen der EB auftreten ( $\bullet$ Abb. $\mathbf{6}$ ).

Die Pigmentläsionen zeigen das gleiche Schicksal wie die gemeinhin erworbenen melanozytären Nävi. Sie treten im ersten oder zweiten Lebensjahrzehnt in Erscheinung und sind dabei zunächst flach, dunkel und wachsen horizontal, um später ihre dermalen Anteile unter Pigmentierungsverlust zu vermehren, womit ein chagrinähnlicher Aspekt analog zu dermalen Nävi ensteht.

Wendet man den klinischen Algorithmus der ABCDE-Regel zur Beurteilung von Pigmentläsionen an, sind EB-Nävi klinisch wie dermatoskopisch meist hochgradig Melanom-verdächtig. Dies gilt insbesondere dann, wenn es - wie bei EB-Nävi nicht unüblich - zu einem manchmal explosiven Wachstum oder zum Auftreten von Satellitenläsionen kommt. Andererseits fehlen dermatoskopisch stahlblaue Areale als Hinweis für Melaninablagerungen innerhalb der papillären Dermis ebenso wie „black dots“ (Pigment im Stratum corneum als Zeichen pagetoiden Aufsteigens von Melanozyten in der Epidermis) oder „blue-whitish veils“ (von Akanthose bedeckte Pigmentaggregationen), somit auflichtmikroskopische Merkmale, die alle als starke Indikatoren für invasive Melanome gelten [4].

EB-Nävi zeigen klinisch Parallelen zum Melanom und sind daher kontrollbedürftig.

Histologische Parallelen zum persistierenden Nävus/Pseudomelanom, einer traumatisierten benignen melanozytären Läsion entsprechend, unterstützen die Hypothese, dass Melanozyten aus inzipienten Nävi oder subklinischen Nävuszellnestern bei der Spaltbildung in der BMZ aus ihrem Verband gelöst werden und sich, nachdem sie zunächst frei in der Blasenflüssigkeit flottieren, schließlich willkürlich (oft auch am Blasenrand) absetzen. Unter Einfluss des wachstumsstimulierenden Mikromilieus einer permanenten epidermalen Regeneration expandieren sie dann klonal. Fortwährende Wundheilungs- bzw. Reparationsprozesse aufgrund chronischer Gewebstraumatisierung mit Zerstörung der Mikroarchitektur, Neovaskularisation und Narbenbildung erklärten damit nicht nur die strukturellen Auffälligkeiten von EB-Nävi, sondern begründen auch Bedenken, dass der chonische Proliferations-Stimulus über die Zeit eine maligne Transformation begünstigen könnte. Dies ist analog zu einem entsprechenden Modell für die Erklärung der gehäuften Entstehung von hoch aggressiven Plattenepithelkarzinomen bei einigen der dermolytischen EBSubtypen. Wiewohl das Patientenkollektiv klein ist, wurde in über 25 Jahren eingehender Beobachtung unsererseits noch kein aus einem EBNävus hervorgegangenes Melanom diagnostiziert [4]. Dementsprechend und auch unter Bedachtnahme auf die Hautfragilität von EB-PatientInnen ist eine konsequente, regelmäßige klinische, dermatoskopische und gegebenenfalls bioptische Untersuchung verdächtig erscheinender Stellen in den durchaus sehr großflächigen EB-Nävi gegenüber einer prophylaktischen Totalexzision vorzuziehen.

\section{EB und Karzinome}

Bereits ab der 2. und 3. Lebensdekade treten vor allem bei Patienten mit REBD, DEBD sowie, zu einem geringeren Anteil, auch der EBJ oft multiple, hoch aggressive und schnell metastasierende Plattenepithelkarzinome auf, die wenig chemound radiosensibel sind ( $\bullet$ Abb. 7).

Dabei beträgt die kummulative Häufigkeit dieser Malignome z.B. für die schwere, generalisierte REBD-Patienten 0,8\%, 7,5\%, 67,8\%, 73,4\%, 80,2\% bzw. 90,1\% im Alter von 14, 20, 35, 40, 45 bzw. 55 Jahren. Diesen folgen die leichteren REBD-Formen und die REBD inversa. Das Risiko für die Entstehung von Plattenepithelkarzinomen korreliert dabei mit dem Schweregrad und der Ausdehnung 
von Ulzerationen und Narben an der Haut. Pathogenetisch scheinen die rezidivierende Gewebetraumatisierung, die BMZ-Barrieredefizienz, eine reparative (Hyper-)Proliferation mit entsprechendem Zytokinmilieu, die Alteration der interzellulären Kohärenz (Kontaktinhibition!) und Störungen der Immunabwehr eine bedeutende Rolle zu spielen.

Die REBD-Varianten sind mit einem sehr hohen Risiko für die frühe Entwicklung von aggressiven Plattenepithelkarzinomen behaftet.

Aufgrund des hochmalignen biologischen Verhaltens dieser Tumore ist deren Früherkennung von entscheidender Bedeutung, dabei aber besonders problematisch. Oft als nicht heilende, mit Krusten belegte Wunden oder Granulationsgewebe imponierend, ist nämlich die Differenzierung der Karzinome von den tumorfreien, teils ausgedehnten Erosionen, Krusten oder Narben nicht nur im Anfangsstadium außerordentlich schwierig.

Wiederholte Biopsien an mehreren Stellen der verdächtigen Läsionen und eine im Bedarfsfall frühe ausgedehnte chirurgische Intervention sind im Fall des Falles entscheidend. Eventuell kann, unter Berücksichtigung des hohen Metastasierungspotenzials, eine Schildwächter-Lymphknotenbiopsie angeschlossen werden. Bei der klinischen Inspektion der Lymphknotenstationen muss bei vergrößerten, tastbaren Lymphknoten differenzialdiagnostisch auch an eine Lymphadenopathie bedingt durch die chronische Hautentzündung und wiederholte Hautinfekte gedacht werden. Chemotherapeutische Regime, die Gabe von systemischen Retinoiden und COX-2-Inhibitoren oder die Radiotherapie von Primärtumoren oder Lymphknotenmetastasen (Restriktion: Hautverletzlichkeit der EB-Patienten) wurden mit fraglicher Effektivität und vorrangig palliativ als Behandlungsmöglichkeiten in der Literatur beschrieben.

\section{Wichtige Komplikationen}

$\nabla$

Neben mukokutanen Veränderungen können im Rahmen der EB teilweise bereits ab dem frühesten Kindesalter zahlreiche Systemmanifestationen auftreten, die einen entscheidenden Einfluss auf die individuelle Prognose der Betroffenen haben und in der Regel einen multidisziplinären therapeutischen Zugang erfordern.

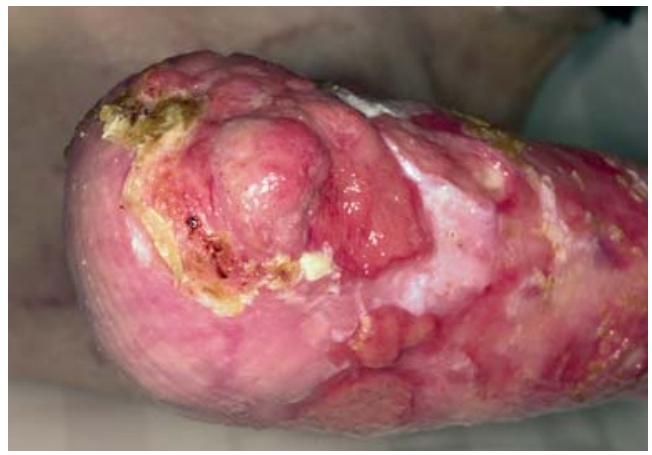

Abb. 7 Mehrere bis eigroße Knoten eines Plattenepithelkarzinoms im Bereich von großflächigen chronischen Wunden am Ellbogen einer Patientin mit schwerer, generalisierter REBD.

Auge

Die Frequenz einer ophthalmologischen Beteiligung variiert nach EB-Subtyp und liegt bei 0,9-6,2\% für die EBS bzw. $40 \%$ und $50 \%$ bei EBJ (EBJ-H) sowie schwerer, generalisierter REBD. Analog zur Pathogenese an der Haut, reichen Augenreiben oder oft nur Blinzeln aus, um friktionsbedingt schmerzhafte Blasen und Erosionen an Kornea, Konjunktiven und Augenlidern zu verursachen. Die in der Nacht allgemein reduzierte Produktion von Tränenflüssigkeit fördert das Anhaften des inneren Lides am Epithel der Kornea, was dann morgens beim abrupten Öffnen des Auges die Gefahr epithelialer Defekte erhöht. Weiterhin umfasst das Symptomen-Spektrum Photophobie, Hornhautnarben, Pannus-, Symblepharon- (bei REBD und EBJ 10\% bzw. 5\%) und Ektropionbildung sowie die Obstruktion des Tränendrüsengangs.

Blasen, Erosionen und narbige Strikturen betreffen auch Kornea und Konjunktiven.

Therapie. Spontane Erosionen heilen in der Regel ohne Narbenbildung innerhalb von wenigen Tagen ab. Um Infektionen zu verhindern, kommen während des Abheilens der epithelialen Defekte prophylaktisch antibiotische Salben zur Anwendung. Symptomatisch werden orale Analgetika, Lubrikanzien, Hyaluronsäure- oder Vit-A-haltige Gele oder künstliche Tränenflüssigkeit, insbesondere über Nacht, verordnet. Um die Photophobie zu reduzieren, empfehlen sich Sonnenbrillen. Friktionsmindernd können auch Augenverbände zur okulären Immobilisation oder leichte Brillengläser mit gepolstertem Steg eingesetzt werden. Bei fortgeschrittener, irreversibler Augenschädigung sind chirurgische Maßnahmen (Pannusentfernung, Symblepharolyse, Ektropiumrekonstruktion, Korneatransplantation, Linsenimplantation) notwendig.

\section{Nase, Ohren und obere Atemwege}

Während bei EBS, DEBD sowie REBD Symptome an den oberen Atemwegen meist nur sporadisch auftreten, ist eine Affektion bei der EBJ häufig.

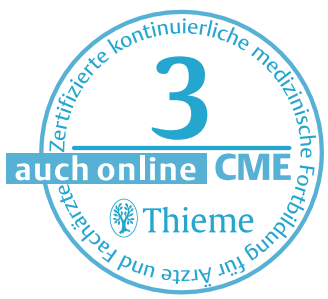




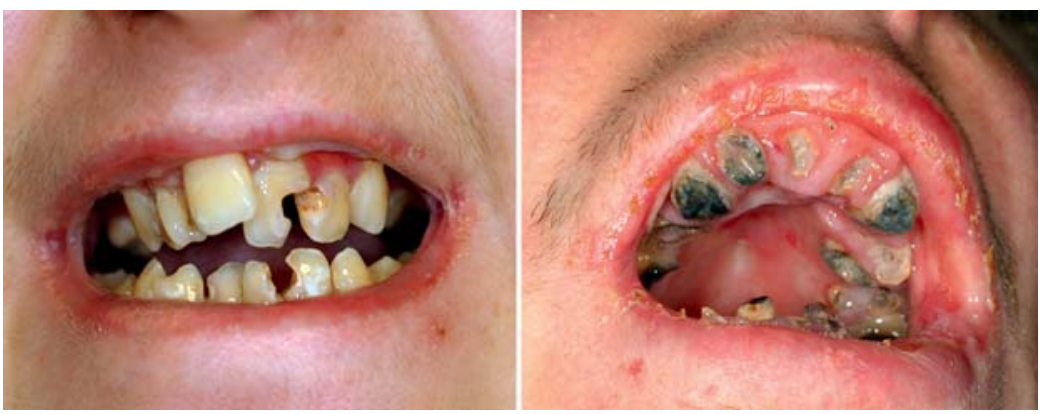

Abb. 8 Mikrostomie, Zahndeformationen und -Fehlstellung, Zahnschmelzdefekte, schwere Karies sowie Peridontitis bei 2 Patienten mit schwerer, generalisierter REBD.

50\% der PatientInnen mit JEB-H und rund 33\% derer mit JEB-O leiden unter Blasen, Ulzerationen, Schleimhautödemen oder -granulationen sowie Vernarbungen mit Destruktion der Stimmbänder oder obliterierenden Stenosen und Strikturen. Klinische Leitsymptome sind chronische Heiserkeit, Dysphonie, inspiratorischer Stridor und akute Dyspnoe.

Da initiale Veränderungen mit entsprechender Symptomatik bereits im Säuglingsalter auftreten können, ist insbesondere bei EBJ-PatientInnen eine frühzeitige und regelmäßige oto-rhino-laryngologische Evaluation und ein Monitoring der tracheolaryngealen Entzündungsaktivität bedeutsam.

Obstruktive Atemwegsveränderungen müssen frühzeitig erkannt und therapiert werden.

Therapie. Die therapeutischen Optionen umfassen unter anderem die Verwendung von Dexamethason- oder Adrenalin-Verneblern sowie Sauerstoff zur Behandlung des Stridors, die Laserdissektion von Strikturen oder Granulationen (bei letzteren wird auch der DNA Synthese-Hemmer Mitomycin C eingesetzt) sowie eine kurative und bei Hochrisikopatienten gegebenenfalls auch elektive Tracheotomie.

Dem gehäuften Auftreten von Otitis externa und media als Folge eines erhöhten Kolonisationsund Infektionsrisikos auf dem Boden einer gestörten epithelialen Barriere bei Patienten mit EB wird mittels regelmäßiger Spülungen mit steriler Kochsalzlösung oder der Gabe antibiotischer und antiseptischer Ohrtropfen begegnet. Die Gefahr einer Resistenzentwicklung bei lang andauernder Anwendung topischer Antibiotika muss allerdings in die Therapieüberlegungen mit einbezogen werden.

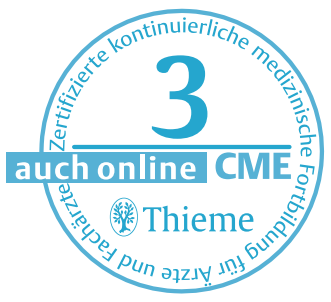

\section{Mundhöhle}

Schmerzhafte Blasen und Erosionen der Mundschleimhaut, die mit erhöhtem Infektrisiko einhergehen und mit Narben, Strikturen und Stenosen abheilen können, sind bei schwerer generalisierter REBD, REBD inversa sowie EBJ-H beson- ders ausgeprägt. Sie behindern auch prophylaktische und therapeutische Bemühungen. In der Folge kommt es bei vielen Patienten zu exzessiver Karies und vorzeitigem Zahnverlust ( $\bullet$ Abb. 8). Postinflammatorische Residuen wie Gebissfehlstellung, Mikrostomie, eine eingeschränkte Mobilität der Zunge und der gestörte Nahrungstransport verschärfen, kombiniert mit einer krankheitsbedingt limitierten manuellen Geschicklichkeit, die Problematik der Nahrungsaufnahme und führen letztendlich zu Mangelernährung, Wachstumsretardierung, Anämie oder gestörter Wundheilung.

Die nahezu ausnahmslos bei Patienten mit EBJ zu beobachtenden Zahnschmelzdefekte (vorrangig Hypoplasien) spiegeln möglicherweise eine, durch defektes Laminin 332 vermittelte, Alteration der Basalmembran in der Anlage des Schmelzorgans während der Odontogenese wider. Die Veränderung könnte analog auch andere epitheliale Strukturen der Mundhöhle von EB-Patienten, beispielsweise den Zahnhalteapparat, betreffen und zu Malokklusion, frühzeitiger Periodontitis und Zahnverlust führen.

Besondere Bedeutung hat die Tatsache, dass ein Milieu chronischer, nicht heilender Ulzerationen und permanenter Reparationsprozesse gemeinsam mit einer funktionellen Änderung von Interzellular- bzw. Zell-Matrix-Kontakten und damit auch der Basalmembran-Barriere das Entstehen von Plattenepithelkarzinomen (ähnlich wie an der Haut) begünstigen und deren Invasionspotenzial steigern kann. Deshalb sind regelmäßige fachärztliche Kontrollen mit Beginn des Zahnens von besonderer Wichtigkeit, um die Möglichkeit zu wahren, Schäden frühzeitig zu erkennen und, so möglich, zu beseitigen.

Prävention und Therapie. Präventive Maßnahmen werden angesichts eines beschränkten therapeutischen Spektrums großgeschrieben und beinhalten z.B. eine konsequente (wenn auch schmerzhafte) Oralhygiene, Fluorid-Substitution, Anwendung nicht alkoholischer Spülungen und die Reduktion einer kariogenen Kost. Füllungen oder rostfreie Stahlkronen kommen zum Schutz des Zahnschmelzes und Aufrechterhaltung der Gebissfunktion zum Einsatz. Bei osteolytischen Herden ist eine Zahnextraktion unumgänglich, um bakterielle Infektionsherde zu vermeiden. Schließlich bieten osseointegrierte Prothesen die Perspektive eines dauerhaften Zahnersatzes unter Umgehung einer signifikanten Schleimhautirritation.

Der besonderen Problematik der EB entsprechend empfiehlt es sich, sämtliche Eingriffe, die Narkosen beinhalten, unter speziellen Bedingungen von mit der Materie vertrauten Spezialisten durchführen zu lassen (Centers of Reference!). Zweckmäßig ist auch eine zeitliche Koordination mehrerer anstehender Eingriffe im Rahmen einer Narkose bzw. Operation. 
Die meist schmerzhafte Beteiligung der Mundschleimhaut führt besonders bei der REBD durch Zahnpflegeprobleme zu exzessiver Karies und Zahnverlust sowie zu Mikrostomie, Dysphagie und Gedeihstörung. Augenmerk muss auch auf die Entstehung von Plattenepithelkarzinomen an der Mundschleimhaut gelegt werden. Die Prävention ist angesichts begrenzter therapeutischer Möglichkeiten von entscheidender Bedeutung.

\section{Gastrointestinaltrakt}

Vor allem bei der schweren, generalisierten REBD, REBD-inversa und EBJ-H ist eine oft allein schon durch feste Nahrung verursachte Traumatisierung des Magen-Darm-Traktes häufig. Schmerzhafte Blasen, Erosionen, Narben, Strikturen und Stenosen lähmen den Willen zur Nahrungsaufnahme und erschweren damit eine adäquate Versorgung mit Nährstoffen, Vitaminen oder Spurenelementen. Gleichzeitig kommt es zur Malabsorption bzw. zum Verlust von Nährstoffen und Vitaminen durch die krankheitsbedingten Veränderungen des Intestinums. Dies alles steht dem erhöhten Energiebedarf durch permanente großflächige Wundheilung, chronische Infektion oder natürliches Wachstum gegenüber. Eine hyperkatabole Stoffwechselbilanz und Mangelerscheinungen sind die Folge.

Als enorale Komplikationen der EB wurden bereits Mikrostomie, Ankyloglossie, eine gestörte Bissfunktion und die Entstehung von Leukoplakien und Plattenepithelkarzinomen angeführt. Im Ösophagus erhöhen proximale Stenosen das Risiko für Aspirationspneumonien, auch Pseudodivertikulose, Barrett-Ösophagus und Perforation wurden als mögliche Komplikationen einer chronischen Beteiligung der Speiseröhre beschrieben. Therapeutisch wird hierfür die endoskopische oder fluoreszenzgeleitete Ballon-Dilatation eingesetzt, bei welcher stärker traumatisierende longitudinale Scherkräfte vermindert und daher bessere Langzeitergebnisse erzielt werden können.

Obstipation. Eine chronische Obstipation stellt das häufigste Symptom einer Magen-Darmtraktbeteiligung bei EB-Patienten dar. Schmerzhafte, zum Stuhlverhalt führende Analfissuren, geringe Zufuhr von Ballaststoffen wegen der multifaktoriell begrenzten Nahrungsmittelaufnahme und exzessiver Flüssigkeitsverlust über die Haut verschärfen die Problematik. Die Gabe oraler Laktulose kann hilfreich sein, um Komplikationen wie Megakolon und Perforation zu verhindern. Parenterale Ernährung und Gastrostomie sind in schweren Fällen von Gedeihstörung die einzige Möglichkeit für eine ausreichende nutritive Versorgung.

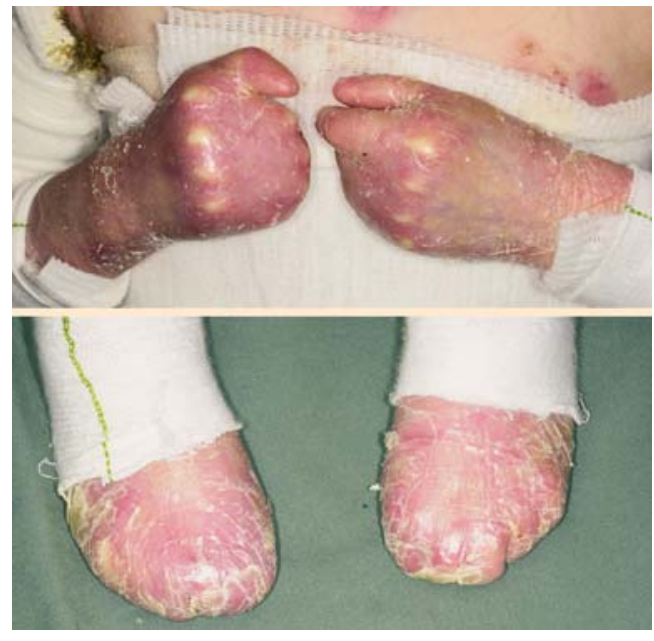

Abb. 9 Pseudosyndaktylien (epidermaler Kokon) mit Hautatrophie, Nagelverlust, Verkürzung der Endphalangen und schweren Gelenkskontrakturen an Händen (oben) und Füßen (unten) einer Patientin mit schwerer, generalisierter REBD.

Vorrangig bei REBD auftretende intestinale Erosionen, Divertikel und Strikturen sind Ursache für Dysphagie, Malabsorption, Obstipation und Dystrophie. Sie bedürfen einer nutritiven Supplementierung und nicht selten chirurgischer Interventionen.

\section{Muskuloskeletale Deformationen}

Die auffälligste, meist bei der schweren generalisierten REBD, REBD-I und seltener auch bei DEBD sowie EBJ auftretende extrakutane Komplikation der EB ist die Pseudosyndaktylie, die bis zum vollständigen Funktionsverlust der Hände und zur Gehunfähigkeit führen kann. Ihre Inzidenz bei Patienten mit schwerer generalisierter REBD beträgt bis zum 6 . Lebensjahr $75 \%$. Die akralen Verwachsungen sind das Resultat ständiger Reparationsprozesse bei permanenter Blasen- und Erosionbildung an (mechanisch besonders exponierten) Händen und Füßen ( $\boldsymbol{O}$ Abb. 9).

Zunächst nur durch partielle Fusion und Synechienbildung gekennzeichnet, kommt es im fortgeschrittenen Stadium zum vollständigen Verschmelzen der Finger sowie Zehen in einem „epidermalen Kokon". Ohne Intervention treten dann rasch Kontrakturen, Muskelatrophie und irreversible Knochenresorption mit bleibenden funktionellen Defiziten auf.

Prävention und Therapie. Da sich Pseudosyndaktylien bei den schweren EB-Subtypen oft bereits in den ersten Lebensjahren entwickeln, ist die frühzeitige Anwendung von speziellen Verbänden und Schienen, die vor Traumatisierung schützen bzw. die Separation der Finger aufrecht erhalten sollen, in Kombination mit spezieller, auch die sensomotorische Fähigkeiten trainierender Physiotherapie von entscheidender präventiver Bedeutung. Dies umso mehr, als dass die operative Lösung der Verwachsungen mit nachfolgender Deckung durch Spalt-, Voll- oder künstliche Haut zwar technisch durchaus möglich ist, der Eingriff aber durch eine hohe Rezidiv- 
rate in seiner Effizienz limitiert wird. Dies macht wiederholte Interventionen (etwa alle 2 Jahre) notwendig, die Kinder sehr oft ablehnen [2].

Pseudosyndaktylien führen insbesondere bei der REBD zum Funktionsverlust von Händen und Gehunfähigkeit. Operative Verfahren sind durch ein hohes Rezidivrisiko gekennzeichnet.

Weitere spezielle internistische Komplikationen

Kardiomegalie. Eine Kardiomegalie tritt insbesondere bei Patienten mit REBD aufgrund des enteropathisch bedingten Mangels an Spurenelementen (Selen, Karnitin, Thiamin), als Folge einer Eisenüberladung durch langjährig wiederholte Transfusion, wegen chronischer Anämie oder nach viralen Infektionen (Myokarditis) auf. Klinische Zeichen für eine Herzinsuffizienz sollten bei den entsprechenden EB-PatientInnen beachtet und durch regelmäßige, gezielte Laborkontrollen überprüft werden.

Renale Manifestationen. Renale Manifestationen wie Dysurie, Hämaturie, Hydronephrose oder Niereninsuffizienz wurden auf dem Boden von narbigen Strikturen, sekundärer Amyloidose oder Glomerulonephritiden, die als Folge von kutanen Streptokokkeninfekten auftreten, vor allem bei den Varianten schwere generalisierte REBD und EBJ-PA berichtet. Letztere wird auch häufig durch Uretrastenosen und -divertikel, Hypo- und Epispadie, Vernarbungen im Bereich des äußeren Genitale oder eine erhöhte urogenitale Infektanfälligkeit verkompliziert. In fortgeschrittenen Fällen helfen nur operative Maßnahmen wie Katheterisierung, Dilatation, Stentimplantation, Elektroresektion, Meatotomie oder Ureterosigmoidostomie.

Osteopenie, Osteoporose. Osteopenie und Osteoporose kommen bei $32 \%$ bzw. $60 \%$ der Patienten mit schwerer generalisierter REBD vor. Als Ursache werden chronische Unterernährung mit Kalziummangel, gleichzeitig bestehende renale Insuffizienz, Immobilisierung und verminderte physische Aktivität gesehen. Therapeutisch vorrangig ist die Behebung von Mangelzuständen.

Chronische Anämie. Die chronische Anämie als Konsequenz von Mangelernährung bei zusätzlicher krankheitsbedingter intestinaler Resorptionsstörung und gleichzeitig permanentem Verlust von Blut, Eisen und Serumprotein über offene Haut- und Schleimhautwunden, äußert sich durch chronische Müdigkeit, Kraftlosigkeit und gestörte Wundheilung. Eine perorale Eisenersatztherapie ist oft durch eine EB-spezifische gastrointestinale Symptomatik unzureichend wirksam. In diesem Fall sind wiederholte Transfusionen notwendig.

Geschlechtsverkehr ist für die meisten Patienten beschwerdefrei durchführbar und auch eine
Schwangerschaft verläuft glücklicherweise in der Regel problemlos. Ein Kaiserschnitt ist beispielsweise nicht routinemäßig notwendig [2].

Breitgefächerte internistische Komplikationen betreffen Herz, Niere, Knochen oder Blut, stehen in direktem Zusammenhang mit der Erkrankung und erfordern ein interdisziplinäres und oft breit gefächertes Behandlungsregime.

\section{Diagnose}

\section{$\nabla$}

Die EB gehört mit einer Prävalenz von 4.6/1000000 und Inzidenz von 10.75/1000000 Lebendgeborener zu den seltenen Erkrankungen („orphan diseases“) [2]. Damit verbunden ist ein Mangel an standardisierten klinischen Daten und diagnostischer Routine außerhalb von spezialisierten Zentren.

Heute erwarten die Eltern eines Neugeborenen mit Blasenbildung die Diagnose berechtigterweise meist sofort nach der Geburt. Die rein klinische Präsentation ist dabei allerdings nur mit Vorbehalt diagnostisch zu werten. Zwar leiden Patienten ohne Vernarbung, Nageldystrophie, Atrophie und Milien sehr wahrscheinlich unter EBS und weisen herpetiform-gruppierte Blasen oder eine ausgeprägte entzündliche Granulation im Gesicht auf EBS-DM bzw. EBJ-H hin. Aber wichtige Leitsymptome wie Milien, Nageldystrophie oder Vernarbung sind zum Zeitpunkt einer (vorläufigen) Diagnosestellung in frühester Kindheit oft noch nicht ausgeprägt. Manche Läsionen können zudem nur transient oder inkonstant in Erscheinung treten. Weiterhin sind auch Spontanrückbildungen (wie beispielsweise von Granulationsgewebe) möglich. So verwundert es nicht, dass retrospektive Analysen von gut dokumentierten Patientenregistern zeigen, dass Sensitivität und Spezifität einer rein klinischen Beurteilung, selbst wenn sie von EB-Experten und unter Kombination von 3 klinischen Zeichen durchgeführt wurde, nie Werte von über $90 \%$ erreichten $[5,6]$.

Bei Blasen- und Erosionsbildung im Neugeborenenalter bilden eine detaillierte Anamnese bezogen auf Patient, Familie, Schwangerschafts- und Krankheitsverlauf, die Bestimmung einer mikrobiellen Kontamination (Gram- und Giemsafärbung, Tzanck-Test, Pilzbefund, bakterieller Abstrich, PCR) und die Entnahme einer Biopsie von klinisch unbefallener, nicht sonnenexponierter Haut (z. B. innerer Oberarm) für Routinehistologie, direkte Immunfluoreszenz, Antigenmapping und Elektronenmikroskopie, gemeinsam mit indirekter Immunfluoreszenz und Mutationsanalysen aus Blutproben einen diagnostischen Algorithmus ( $\bullet$ Tab. 2).

Die Routinehistologie mit Hämatoxilin-EosinFärbung erlaubt lediglich die Unterscheidung 
Tab.2 Auswahl spezieller diagnostischer Möglichkeiten bei EB.

\begin{tabular}{|c|c|c|c|}
\hline Untersuchungstechnik & Hauptgruppe & Untergruppe & Diagnostische Charakteristika \\
\hline \multirow[t]{3}{*}{ Routinelichtmikroskopie } & EBS & EBS basal & Intraepidermale Zytolyse von basalen Keratinozyten \\
\hline & & EBS suprabasal & $\begin{array}{l}\text { Intraepidermale Zytolyse von suprabasalen Keratino- } \\
\text { zyten, Spaltbildung zwischen Stratum corneum und } \\
\text { granulosum }\end{array}$ \\
\hline & $\begin{array}{l}\text { EBJ u. } \\
\text { EBD }\end{array}$ & & „Subepidermale“ Spaltbildung \\
\hline \multirow[t]{10}{*}{ Elektronenmikroskopie } & EBS & & $\begin{array}{l}\text { Intrazytoplasmatische Vakuolenbildung mit Zytolyse der } \\
\text { (supra-)basalen Keratinozyten }\end{array}$ \\
\hline & & EBS-DM & Verklumpung der Keratinfilamente \\
\hline & & EBS-MD & $\begin{array}{l}\text { Dissoziation der Keratinfilamente von Hemidesmosomen, } \\
\text { Fehlen intrazellulärer Plaques }\end{array}$ \\
\hline & & EBS-AR & $\begin{array}{l}\text { Fehlende oder reduzierte Keratinfilamente in basalen } \\
\text { Keratinozyten }\end{array}$ \\
\hline & & EBS-PA & $\begin{array}{l}\text { Reduzierte Integration von Keratinfilamenten in Hemi- } \\
\text { desmosomen }\end{array}$ \\
\hline & EB] & & $\begin{array}{l}\text { Spaltbildung innerhalb der Lamina lucida, } \\
\text { hypoplastische/reduzierte Hemidesmosomen }\end{array}$ \\
\hline & EBD & & Kohärenztrennung unterhalb der Lamina densa \\
\hline & & $\begin{array}{l}\text { Schwere generalisierte } \\
\text { REBD }\end{array}$ & Ankerfibrillen fehlend \\
\hline & & Generalisierte REBD & Ankerfibrillen morphologisch verändert \\
\hline & & DEBD & $\begin{array}{l}\text { Ankerfibrillen morphologisch normal, aber in Anzahl } \\
\text { reduziert }\end{array}$ \\
\hline \multirow[t]{11}{*}{ Antigenmapping } & EBS & & $\begin{array}{l}\text { Semiquantitative Beurteilung der defekten Expression } \\
\text { von Keratin } 5 \text { und } 14\end{array}$ \\
\hline & & EBS-MD & Plektin fehlend oder reduziert \\
\hline & & EBS-PA & $\alpha 6 \beta 4$ Integrin fehlend oder reduziert \\
\hline & EB] & & $\begin{array}{l}\text { Typ-IV-Kollagen der Lamina densa auf der dermalen Seite } \\
\text { (Blasenboden) der Spaltbildung }\end{array}$ \\
\hline & & EBJ-H & Verlust oder Reduktion von Laminin 332 \\
\hline & & $E B J-P A$ & $\begin{array}{l}\text { Fehlende immunhistochemische Färbung mit anti- } \alpha 6 \text { - } \\
\text { und/oder anti- } \beta 4 \text {-Integrin-Antikörpern }\end{array}$ \\
\hline & & $\mathrm{EBJ}-\mathrm{nH}$ & $\begin{array}{l}\text { Reduzierte oder fehlende Expression von Laminin } 322 \text { oder } \\
\text { BPAG2/Typ-XVII-Kollagen }\end{array}$ \\
\hline & EBD & & $\begin{array}{l}\text { Typ-IV-Kollagen der Lamina densa auf der epidermalen } \\
\text { Seite (Blasendach) der Spaltbildung }\end{array}$ \\
\hline & & $\begin{array}{l}\text { Schwere generalisierte } \\
\text { REBD }\end{array}$ & $\begin{array}{l}\text { Bei schwerem Verlauf fehlendes oder massiv vermindertes } \\
\text { Typ-VII-Kollagen }\end{array}$ \\
\hline & & DEBD & Normales Muster von Typ-VII-Kollagen \\
\hline & Kindler & & Kindlin-1 fehlend oder reduziert \\
\hline
\end{tabular}

zwischen intraepidermaler und subepidermaler (kann junktional oder dermolytisch sein) Blasenbildung und ist daher von begrenztem praktischen Wert [2].

Transmissions-Elektronenmikroskopie.

Die Transmissions-Elektronenmikroskopie (TEM) und das Immunfluoreszenz-Antigenmapping ermöglichen die Bestimmung der diagnostischen Spaltebene in EB-Haut (intraepidermal, in der Lamina lucida oder unter der Lamina densa). Mithilfe der TEM können zudem die bei den jeweiligen EB-Subtypen pathognomonisch veränderten Strukturen (oder deren Fehlen) wie Keratinfilamente, Hemidesmosomen oder Ankerfibrillen beurteilt und semiquantitativ erfasst werden. Die Technik und deren diagnostische Interpretation die EB betreffend ist jedoch sehr anspruchsvoll und auch teuer und sollte Referenzlaboratorien mit entsprechender Expertise vorbehalten bleiben [2].
Antigen-Mapping. Bedeutend einfacher, kosteneffizienter sowie rasch durchführbar ist das Immunfluoreszenz-Antigen-Mapping [7]. Hierbei bestimmt man einerseits die Spaltebene mittels des Nachweises eines Markerproteins (z. B. Typ-VIIKollagen) in Bezug auf dessen Lokalisation in der Blasenbiopsie ( $\bullet$ Abb. 10).

Die Methode lässt andererseits auch Rückschlüsse auf die Expression (normal - reduziert - fehlend) der Schlüssel-Stukturproteine der BMZ zu. Damit werden auch die Kandidatengene bestimmt, die dann mit bedeutend aufwendigeren Mutationsanalysen weiter untersucht werden. Allerdings ist zu bedenken, dass rein funktionelle Änderungen von Strukturproteinen mit einem normalen Antigenfärbemuster einhergehen können [2].

Mutationsanalysen. Mutationsanalysen (Heteroduplexanalyse, denaturierende Hochdruckflüssigkeitschromatografie, Restriktionsendonukleasenverdau, allelspezifische Oligonukleotidhybri-

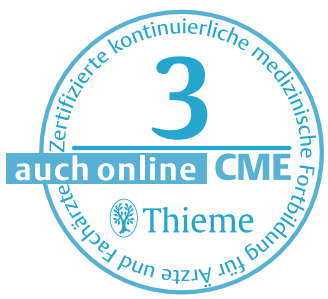



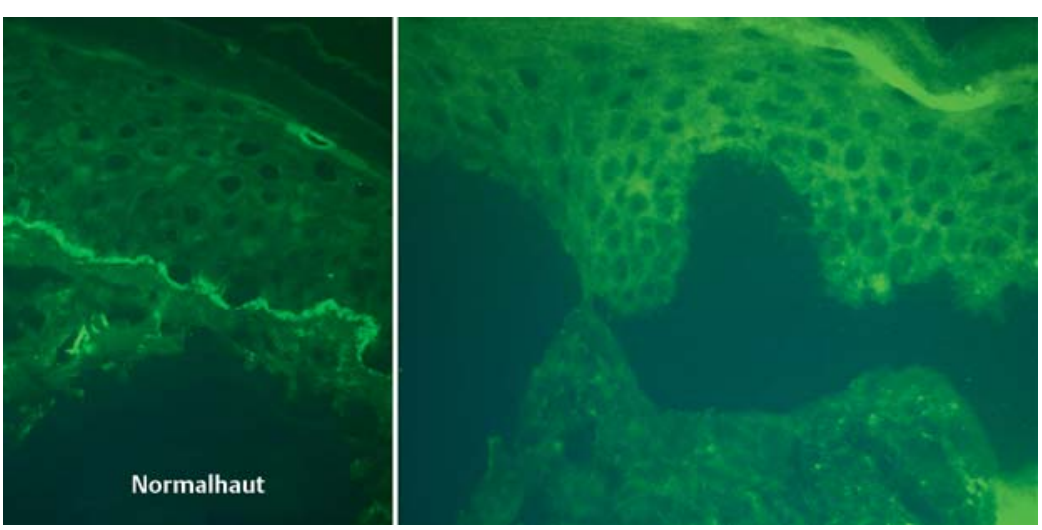

Abb. 10 Antigenmapping. Im Vergleich zur positiven Normalhautkontrolle (links) mit regelrecht exprimiertem Typ-XVII-Kollagen (BPAG-2) in der dermo-epidermalen BMZ zeigt die Haut eines Patienten mit generalisierter EBJ-non-Herlitz Blasenbildung und eine vollständig fehlende Reaktivität (rechts).

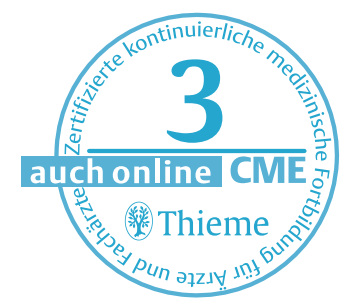

disierung bzw. direkte Gensequenzierung) erlauben die präzise Bestimmung von Art und Lokalisation molekularer Veränderungen und stellen damit die Grundlage für eine genetische Beratung und spezifische gentherapeutische Interventionen dar [2]. Zudem ist diese Untersuchung heute Basis für die Pränatal- (Chorionzottenbiopsie, Amniozenteseproben) und - sofern gesetzlich erlaubt - Präimplantations-Diagnostik [2]. Allerdings sind Genotyp-Phänotyp-Korrelationen teils beträchtlichen Schwankungen unterlegen, sodass Mutationsanalysen als primäres Verfahren zur Subklassifizierung von Patienten ohne Familienanamnese ungeeignet erscheinen.

Die Diagnose der jeweiligen EB-Hauptgruppen erfolgt mittels Antigen-Mapping oder Transmissions-Elektronenmikroskopie. Eine Subklassifizierung gelingt dann durch eine Zusammenschau von Familienanamnese, klinischer Präsentation und Labordaten. Mutationsanalysen erlauben die präzise Genotypisierung und damit Klassifizierung und dienen einer Pränatal- oder Präimplantationsdiagnostik sowie vorrangig auch Forschungszwecken.

\section{Therapie}

Dauernde Blasenbildung, chronische Entzündung, Barrieredefizienz und damit eine verstärkte mikrobielle Kolonisation mit verschiedenen Keimen und häufigen Infekten (Sepsisgefahr!), schlechter Ernährungszustand und die reduzierte Sauerstoffversorgung lassen die Hautläsionen der EB zu chronischen, schlecht heilenden und oft sehr schmerzhaften Wunden werden. Die Wundheilungsstörungen spiegeln letztendlich die integrale Funktion von Strukturproteinen der BMZ in der Regulation von kutanen Reparationsprozessen, wie beispielsweise der Keratinozytenmigration und BMZ-Regeneration, wider.
Prävention. Es bedarf einer vielschichtigen Strategie, genannten Komplikationen therapeutisch zu begegnen. Am Beginn steht die Prävention der Blasenentstehung durch kühle Umgebungstemperatur, Druckentlastung der Haut unter Verwendung von Wasser- oder Luftmatrazen und weichen Sitzpolstern oder geschmeidiger, nicht irritativer sowie einfach anzuziehender Kleidung (z.B. Baumwolle, Seide) ohne reibende Oberflächen (Nähte, Etiketten).

Verbände. Offene Hautstellen werden mit antiseptischen bzw. antimikrobiellen, atraumatischen nicht adhäsiven Funktionsverbänden, die auch Schutz vor mechanischer Exposition bieten, versorgt. Beim Verbandswechsel bietet sich an, die Verbände lediglich aufzuschneiden und durch ein Bad mit einem Antiseptikumzusatz abzulösen ( $\odot$ Abb. 11).

Schmerz. Akuter (Blasen, Erosionen, gastrointestinaler Reflux, Ösophagusstenosen oder -spasmen, Zahnerkrankungen, besonders auch Erosionen der Kornea), chronischer (persistierende Entzündung der Haut, neuropathische und Knochenschmerzen, Obstipation, Kontrakturen) oder prozeduraler Schmerz (Verbandswechsel, Bäder) bedarf einer angepassten pharmakologischen und psychologischen Analgesie [2]. Präventiv wirken dabei Physio- und Hydrotherapie, weiche Kost bei Dysphagie und Mundschleimhautbefall oder Laxantien und ballaststoffreiche Kost bei Obstipation, Analfissuren und perianalen Ulzerationen.

Juckreiz. Wundheilungsprozesse, die kontinuierliche inflammatorische Aktivität mit Stimulation von Mastzellen und Hauttrockenheit sind vermutlich die Ursache des insbesondere bei PatientInnen mit EBS-DM, DEBD und speziell den „pruriginösen“ Formen oft ausgeprägten, quälenden Juckreizes [2]. Reaktives Reiben und Kratzen führt durch Freisetzung von Entzündungsmediatoren zu einem Circulus vitiosus. Maßnahmen bei zu trockener Haut (wie Flüssigkeitsaufnahme, Aufenthalt in nicht zu heißem und trockenem Raumklima, Vermeidung dehydrierender alkoholischer Lösungen oder Seifen), Hautkühlung (kalte Kompressen, kühlende Kleidung) und das Unterbinden genannten Circulus vitiosus (Juckreiz-Kratzen - mehr Juckreiz) durch Kürzen der Fingernägel, Tragen von Baumwollhandschuhen oder Entspannungsübungen sind wichtige Ansätze zur Reduktion des Pruritus. Symptomatisch können topische oder systemische Kortikosteroide und Tacrolimus eingesetzt werden. Antihistaminika sind oft mit Ausnahme des sedierenden Effektes von H1-Antihistaminika der ersten Generation wirkungslos. Fallberichte zur Anwendung von niedrigdosiertem Cyclosporin A, Dapson oder oralem Thalidomid sind bekannt, das Nebenwirkungsprofil dieser Medikamente ist in die Therapieentscheidung allerdings mit einzubeziehen. 
Hyperkataboler Status. Der permanente Verlust von Blut, Serum, Proteinen und Wärme über erosive Hautareale, eine chronische inflammatorische Aktivität sowie die eine Nahrungsaufnahme und -resorption limitierenden oralen, pharyngealen, ösophagealen und anderen gastrointestinalen Komplikationen der EB führen zu einem hyperkatabolen Status, der eine adäquate Substitution mit Nährstoffen und Vitaminen/Spurenelementen erfordert. Diese für die Entwicklung der Patienten so wichtige Maßnahme gelingt meist nur unter Einbeziehung einer professionellen Ernährungsberatung und durch regelmäßiges Labormonitoring [2].

Prophylaktische und therapeutische Bemühungen umfassen die Prävention einer Traumatisierung, optimierte Wundversorgung durch Funktionsverbände, die symptomatische Therapie von z. B. Schmerz und Juckreiz, eine adäquate Nährstoffzufuhr sowie im Bedarfsfall ein operatives Vorgehen.

\section{Gentherapie}

Angesichts der Multimorbidität der EB-PatientInnen bleibt der kurative Therapieansatz das eigentliche Ziel [2]. Tatsächlich ist als Proof of Principle 2005 erstmals bei einem EB-Patienten die Gentherapie gelungen [8]. Dabei wurden EBJ-nH Keratinozyten mit molekular korrigiertem LAMB3 transduziert. Diese in Kultur gezüchteten Zellen wurden dann auf die mit einem Laser vorbereiteten Oberschenkelareale des Patienten übertragen, wo dann eine adäquate Synthese und Aggregation von Laminin 332 mit nachhaltiger Bildung einer festhaftenden und sich selbst erneuernden Epidermis zu beobachten war. Ein wichtiger Punkt war, dass die beim Patienten nachgewiesene geringe endogene Restproteinproduktion wohl eine Autoimmunreaktion verhinderte.

Trotzdem ist der letzte Schritt zu einer individualisierten, spezifischen, für alle zugänglichen gentherapeutischen Intervention noch nicht vollzogen. Dabei profitieren molekulartherapeutische Ansätze bei Genodermatosen von der leichten Zugänglichkeit des Zielorgans, der Kultivierbarkeit von Keratinozyten und der unmittelbaren, nicht invasiven Visualisierbarkeit des Erfolges (wie auch der Nebenwirkungen mit z.B. der Möglichkeit der sofortigen Entfernung des Transplantates). Während bei rezessiv vererbten Varianten die Einführung eines genetisch korrigierten Alleles mittels viraler oder non-viraler Vektoren zur Expression eines intakten Proteins für eine phänotypische Korrektur ausreichen kann, ist bei dominanten Mutationen das aberrante Allel zu neutralisieren oder zu reparieren. Hierfür eignen sich Biotechnologien wie Antisense/OligonukleotidStrategien, Ribozym-Therapie, RNA-Interferenz oder trans-splicing [9]. Der Transfer von thera-

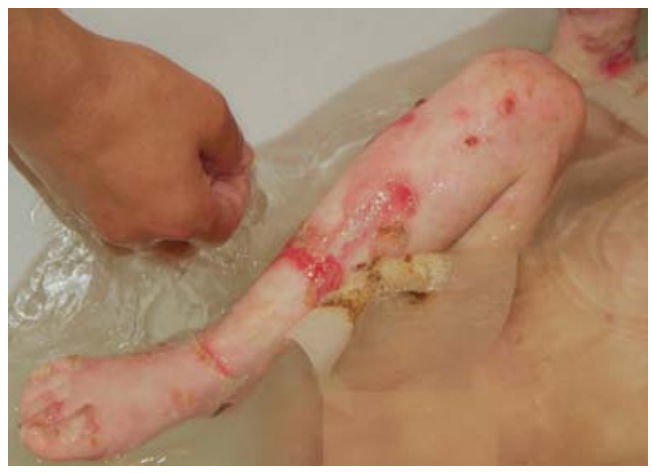

Abb. 11 Lösen von Verbänden bei einem Vollbad bei einem Patienten mit generalisierter EBJ-non-Herlitz.

peutischen Genen erfolgt entweder direkt (in vivo) in die Patientenhaut oder ex vivo z.B. in Zellkultur mit anschließender Retransplantation. Zur nachhaltigen Korrektur der Erkrankung braucht es unter anderem die Identifikation und Selektion von Stammzellen und den effizienten wie praktikablen Transfer von therapeutischen Genen oder Regulatoreinheiten in Zielzellen, durch die epidermale Barriere. Weitere Probleme sind das Risiko der Insertionsmutagenese oder Dysregulation lokaler Regulationsmechanismen durch eine genomische Integration von therapeutischen Genen, die Immunogenität des viralen Vektors bei in-vivo Strategien, die Antigenität des neu eingebrachten, dem Körper unbekannten Proteins (inbesondere bei wiederholter Administration mit möglicher Induktion bullöser Autoimmunerkrankungen), das Infektionsrisiko oder die zu erwartenden Wundheilungsstörungen im Rahmen chirugischer Ex-vivo-Therapien.

Bei zahlreichen anderen molekulargenetischen Therapieprojekten konnte unter anderem gezeigt werden, dass die intradermale Injektion von COL7A1-korrigierten, autologen Fibroblasten bei Patienten mit schwerer, generalisierter REBD zu einer lokalisationsspezifischen In-vivo-Expression von Typ-VII-Kollagen und phänotypischer Korrektur führte [10]. Eine noch praktikablere therapeutische Perspektive eröffnen weitere Versuche von Woodley [11], nach welchen sich auch intravenös injizierte, autologe, gentechnisch korrigierte EBD-Fibroblasten spezifisch im Wundgebiet anreichern und dieses mit dem Transgen-Produkt so suffizient versorgen, dass funktionelle Verankerungsstrukturen gebildet werden, die ihrerseits die Wundheilung beschleunigen. Konsequenterweise wäre der ultimative Therapieansatz jedoch die Generierung genetisch korrigierter Knochenmarksstammzellen, die eine permanente und den ganzen Körper umfassende Gentherapie ermöglichen würden.

Fortschritte auf dem Gebiet der molekularbiologischen Forschung und die erste gelungene Gentherapie eines EB-Patienten als Proof of Principle lassen die Perspektive einer breit zugänglichen kurativen gentherapeutischen Intervention realistischer erscheinen.

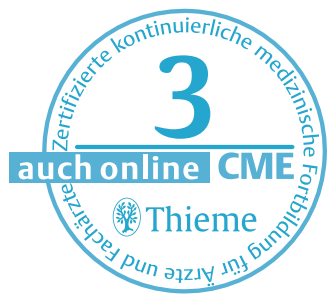




\section{Zusammenfassung}

Die Multimorbidität der EB erfordert zur Betreuung von Patienten einen multidisziplinären $\mathrm{Zu}$ gang und speziell geschulte Experten, idealerweise räumlich konzentriert. In Österreich wurde zu eben diesem Zweck das eb-Haus Austria verwirklicht, das mit seinen 3 Organisationseinheiten (Ambulanz zur multidisziplinären medizinischen Betreuung durch speziell ausgebildete Ärzte und Krankenschwestern, Forschungslabor für die molekulare Diagnostik sowie Gentherapieforschung und EB-Akademie zur kontinuierlichen Aus- und Weiterbildung von Betroffenen, Angehörigen, Laien und medizinischen Fachkräften) dem Ideal einer umfassenden und individualisierten Versorgung von EB-Patienten näherzukommen versucht [2]. Ähnliche Institutionen stehen EB-PatientInnen auch in anderen europäischen Ländern zur Verfügung (www.internationalebforum.org).

\section{Literatur}

1 Fine JD, Eady RA, Bauer EA et al. The classification of inherited epidermolysis bullosa (EB): Report of the Third International Consensus Meeting on Diagnosis and Classification of EB. J Am Acad Dermatol. 2008; 58: 931 - 950

2 Fine JD, Hintner $H$ (eds). Life with Epidermolysis Bullosa (EB): Etiology, diagnosis, multidisciplinary care and therapy. Springer, Wien: 2008

3 Mellerio JE, Pulkkinen L, McMillan JR et al. Pyloric atresiajunctional epidermolysis bullosa syndrome: mutations in the integrin beta4 gene (ITGB4) in two unrelated patients with mild disease. Br J Dermatol 1998; 139: 862 871

4 Lanschuetzer CM, Emberger M, Laimer $M$ et al. Epidermolysis bullosa naevi reveal a distinctive dermoscopic pattern. Br J Dermatol 2005; 153: 97-102

5 Fine JD, Johnson $L B$, Suchindran $C$ et al. Cutaneous and skin-associated musculoskeletal manifestations of inherited EB: the National Epidermolysis Bullosa Registry Experience. In: Fine JD, Bauer EA, McGuire J, Moshell A (eds): Epidermolysis bullosa: clinical, epidemiologic, and laboratory advances, and the findings of the National Epidermolysis Bullosa Registry. Baltimore: Johns Hopkins University Press, 1999

6 Hintner $H$, Wolff $K$. Generalized atrophic benign epidermolysis bullosa. Arch Dermatol 1982; 118: 375-384

7 Hintner H, Stingl G, Schuler G et al. Immunofluorescence mapping of antigenic determinants within the dermalepidermal junction in the mechanobullous diseases. Invest Dermatol 1981; 76: 113-118

8 Mavilio F, Pellegrini G, Ferrari S et al. Correction of junctional epidermolysis bullosa by transplantation of genetically modified epidermal stem cells. Nat Med 2006; 12 : $1397-1402$

9 Bauer JW, Laimer $M$. Gene therapy of epidermolysis bullosa. Expert Opin Biol Ther 2004; 4: 1435-1443

10 Woodley DT, Keen DR, Atha T et al. Injection of recombinant human type VII collagen restores collagen function in dystrophic Epidermolysis bullosa. Nat Med 2004; 10: 693-695

11 Woodley DT, Remington J, Huang Y et al. Intravenoulsy injected human fibroblasts home to skin wounds, deliver type VII collagen, and promote wound healing. Mol Ther 2007; 15: 628-635

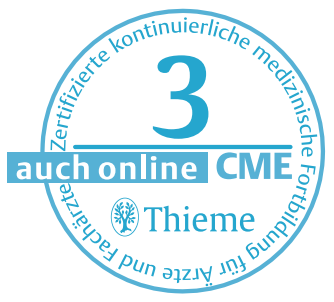




\section{CME-Fragen Epidermolysis bullosa hereditaria}

1 Welches Strukturprotein spielt bei keiner der EB-Varianten eine Rolle?

Desmoglein 1

Typ-XVII-Kollagen

Typ-VII-Kollagen

Plektin

E Keratin 5

2 Wo findet Spaltbildung bei den Hauptgruppen der EB nicht statt?

epidermal

unterhalb der Lamina densa

junktional

desmosomal

dermal

\section{3}

A Sie kann lokal oder generalisiert auftreten.

B Extrakutanmanifestationen sind möglich.

C Nur die EBS-DM hat eine erhöhte Mortalität.

D Homozygote Mutationen sind klinisch weniger problematisch als heterozygote.

E Bei der EB-MD tritt die Muskeldystrophie meist erst verzögert auf.

4 Welche Aussage trifft für die EBJ zu?

1 Periorifizielle Granulationen sind bei EBJ-H häufig. 2 Der EBJ-H liegen homozygote Mutationen zugrunde.

3 Extrakutanmanifestationen sind häufig und schwerwiegend.

4 Schmelzhypoplasien kommen nur bei EBJ-H vor.

5 Die EBJ-nH ist die häufigste EB-Variante.

A Keine Aussage ist richtig.

B Alle Antworten sind richtig.

C Die Antworten 1, 2, 3 sind richtig.

D Die Antworten 2, 3, 4 sind richtig.

E Die Antworten 1, 2, 5 sind richtig.

\section{5}

Für die dominante EBD ist nicht typisch, dass

A sie zu atropher Narbenbildung führt.

B sie familienspezifische Mutationen aufweist.

C eine Poikilodermie auftritt.

D ihr Missense-Mutationen zugrunde liegen.

E ihr Nonsense-Mutationen zugrunde liegen.

\section{6}

A Sie treten am häufigsten bei DEBD auf.

B Sie sind Vorläufer-Läsionen für das Melanom.

C Eine Totalexzision sollte nach Möglichkeit angestrebt werden.

D Sie haben oft klinische und dermatoskopische Zeichen eines Melanoms.

E Sie entsprechen einer postinflammatorischen Hyperpigmentierung.

7 Welches klinische Zeichen lässt Sie am ehesten nicht an eine Komplikation einer schweren Form der EB (mit Systembeteiligung) denken?

A plötzlich auftretende starke Augenschmerzen

B chronische Heiserkeit und/oder Stridor

C akute Dyspnoe

D Dysurie

E Arthralgien

8 Welche ist keine etablierte Komplikation der EB als Systemerkrankung?

Aspirationspneumonie

B Eisenüberladung nach multiplen Transfusionen

C Rachitis

D chronische Anämie

E infektbedingte Myokarditis

9

A Die klinische Präsentation ist nur eingeschränkt wegweisend.

B Die Routinehistologie erlaubt eine klare Unterscheidung zwischen EBS, EBJ und EBD.

C Die Biopsie zur routinehistologischen Beurteilung sollte an klinisch unbefallener, nicht sonnenexponierter Haut erfolgen.

D Das Antigen-Mapping erlaubt eine Beurteilung der Expression und Verteilung sowie eine Funktionsdiagnostik von Strukturproteinen.

E Das Antigen-Mapping wird zur Bestätigung des molekularanalytischen Befundes durchgeführt.

Welches diagnostische Verfahren findet bei Verdacht auf das Vorliegen einer EB keine Anwendung?
A Anamnese
B Tzanck-Test
C ELISA
D direkte Immunfluoreszenz
E Elektronenmikroskopie 